\title{
1 Changing the Waddington landscape to control mesendoderm competence
}

2 James R. Valcourt ${ }^{1,2,3, \dagger}$, Roya Huang ${ }^{2,3}$, Sharmistha Kundu ${ }^{4,5,6}$, Divya Venkatasubramanian ${ }^{2,3}$,

3 Robert E. Kingston ${ }^{4,5}$, Sharad Ramanathan ${ }^{2,3,7,8, \dagger}$

$4{ }^{1}$ Systems, Synthetic, and Quantitative Biology Program, Harvard University, Cambridge, MA

502138

62 Department of Molecular and Cellular Biology, Harvard University, Cambridge, MA 02138,

7 USA

$8 \quad{ }^{3}$ Division of Applied Physics, Harvard University, Cambridge, MA 02138

$9{ }^{4}$ Department of Molecular Biology, Massachusetts General Hospital, Boston, MA, USA

$10{ }^{5}$ Department of Genetics, Harvard Medical School, Boston, MA, USA

$11{ }^{6}$ Present address: Intellia Therapeutics, Cambridge, MA 02139

$12{ }^{7}$ School of Engineering and Applied Sciences, Harvard University, Cambridge, MA 02138, 13 USA

$14{ }^{8}$ Department of Stem Cell and Regenerative Biology, Harvard University, Cambridge, MA

15 02138, USA

$16 \dagger$ To whom correspondence should be addressed. Email: valcourt@g.harvard.edu,

17 sharad@,cgr.harvard.edu

19 Abstract

20 As pluripotent human embryonic stem cells progress towards one germ layer fate, they lose the

21 ability to adopt alternative fates. It is unknown how the cells' competence for these alternative

22 fates changes along their developmental trajectory or if this competence can be modulated. Here,

23 we show that a differentiating stem cell's probability of adopting a mesendodermal fate when

24 given the appropriate signal falls sharply at a specific point along the ectodermal trajectory, and

25 we further demonstrate that this point can be moved using genetic perturbations. Using a low-

26 dimensional reaction coordinate to monitor progression towards ectoderm, we can determine the

27 probability that individual cells at different points along this path can transition to the 
mesendodermal fate upon BMP4 and Activin A signal exposure. Knowing this probability allows

29 us to prospectively isolate and profile differentiating cells based on their mesendoderm

30 competence. Analysis and validation of these RNA-seq and ATAC-seq profiles identified

31 transcription factors that can independently control the cell's mesendoderm competence and its

32 progression along the ectodermal developmental trajectory. In the classical picture of a

33 Waddington landscape, these effects correspond to altering the barrier between fates and changing

34 the cell's location on the landscape, respectively. The ability of the underlying gene regulatory

35 network to modulate these two aspects of the developmental landscape could allow separate

36 control of the dynamics of differentiation and tissue size proportions.

\section{Main}

38 Pluripotent cells have the ability to produce any of the myriad cell types seen in the adult body ${ }^{1}$,

39 but they lose this potential as they differentiate. During initial lineage specification, cells can

40 change their fate choice upon exposure to signals that induce an alternative selection ${ }^{1-4}$, such as

41 by transplantation to a different location in the embryo. In time, however, the cell's fate becomes

42 determined, and it is no longer competent to choose a different lineage in response to the same

43 external signals ${ }^{5-7}$. While lineage specification is relatively well-understood ${ }^{8-17}$, whether and how

44 competence for adopting alternative lineages can be tuned during differentiation is not.

45 Determining how this competence is set and modulated is essential for understanding

46 developmental patterning and plasticity.

47 There is a fundamental challenge in understanding the competence of a cell to choose a specific

48 fate in response to a signal because a cell's fate choice is evident only after the expression of fate- 
49 specific marker genes. In mouse and humans, the fate markers for the germ layers are not expressed

50 until at least twelve hours after exposure to the appropriate signals ${ }^{20-22}$. Since the gene expression

51 and epigenetic state of the cell changes substantially during this period, determining how the

52 molecular state of the cell at the time of signal exposure governs its competence to adopt alternative

53 fates has been difficult.

54 To overcome this challenge, we use a framework motivated by the Waddington landscape ${ }^{23}$. In

55 this picture, the pluripotent cell moves down into the valley corresponding to its chosen fate, and

56 it is prevented from adopting the alternative fate by a barrier that rises between the valleys (Figure

57 1a). Thus, a cell's ability to transition to the alternative fate depends both on its location along the

58 developmental trajectory and the size of the barrier at that point. Using the germ layer selection of

59 human embryonic stem cells (hESCs) as a model system, we measured the probability that a given

60 cell can transition to the mesendoderm fate as a function of its position along the ectodermal

61 developmental trajectory, thereby probing the location of the barrier between fates. Using this

62 probability distribution, we could prospectively isolate and characterize cells based on their

63 mesendoderm competence. Computational analysis of both the gene expression and epigenetic

64 profiles of these populations allowed us to identify candidate genes that we predicted to control

65 mesendoderm competence. By perturbing the levels of these genes, we were able to independently

66 tune the barrier between fates and the cell's position on the Waddington landscape. These dual

67 mechanisms could play a role in regulating the proportions of the different germ layers in the

68 developing embryo. 
bioRxiv preprint doi: https://doi.org/10.1101/867010; this version posted March 14, 2020. The copyright holder for this preprint (which was not certified by peer review) is the author/funder. All rights reserved. No reuse allowed without permission.

a

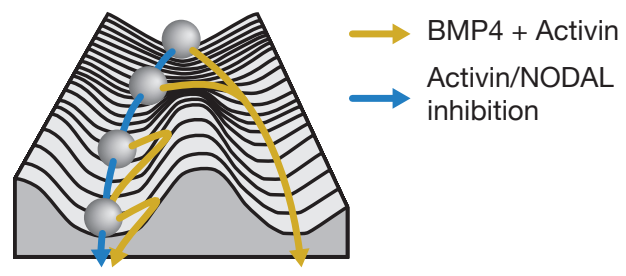

ectoderm mesendoderm

(1) cell location

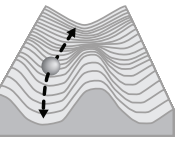

(2) barrier location

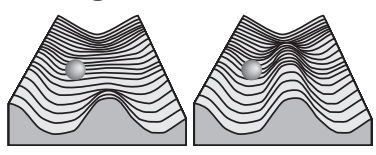

b

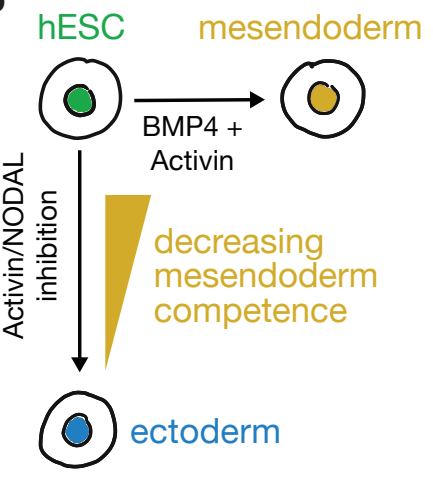

c

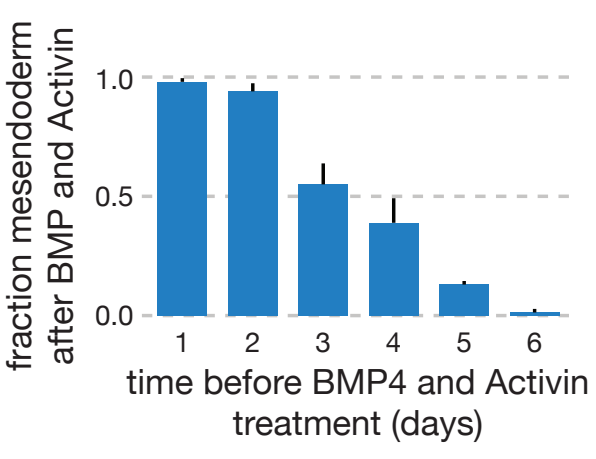

Figure 1 
Figure 1: Stem cells lose competence to adopt mesendodermal fates upon BMP4 and Activin A signal exposure with increasing duration of Activin/NODAL inhibition.

a, Schematic of a Waddington landscape illustrating the ectoderm (blue) / mesendoderm (yellow) fate choice. In this picture, the competence of a cell to produce mesendoderm depends both on the cell's location along the ectodermal developmental trajectory and on the position of the barrier between the ectoderm and mesendoderm fates. b, hESCs choose the ectodermal lineage in response to Activin/NODAL inhibition and mesendodermal lineages in response to BMP4 + Activin A. c, Bar graph of the fraction of cells adopting mesendodermal fate as a function of the duration of ectodermal differentiation after which BMP4/Activin A was added (see Methods). At the population level, with an increasing duration of prior ectodermal differentiation, the competence to choose mesendodermal fates in response to signal decreases. Error bars: std $(n=3)$. 


\section{Results}

70 hESCs lose mesendoderm competence along the ectoderm developmental trajectory

71 Given the appropriate signals, hESCs in vitro can adopt either mesendodermal or ectodermal

72 progenitor fates: BMP4 and Activin/NODAL signals induce mesendodermal fate ${ }^{24}$, while

73 Activin/NODAL inhibition promotes ectodermal fate ${ }^{21}$ (Figure 1b). When cultured in conditions

74 that allow the signaling ligands to access their corresponding receptors on the cells' basolateral

75 side (see Methods), hESCs exposed to BMP4 and Activin A uniformly produce cells expressing

76 BRACHYURY, SOX17, and other markers associated with mesendoderm-derived cell types

77 (Figure 1b, S1a, S1b). In contrast, inhibiting Activin/NODAL signaling promoted ectoderm-

78 derived fates ${ }^{21}$, ultimately producing PAX6 ${ }^{+}$neurectoderm after 4-5 days (Figure 1b, S1c, S1d).

79 Consistent with previous data from mouse 22,25 , we demonstrated that competence to adopt

80 mesendoderm-derived fates decreases at the population level as cells differentiate towards the

81 ectodermal fate. Increasing the duration of differentiation towards ectoderm reduced the fraction

82 of cells that adopted mesendodermal fates in response to BMP4 and Activin A signal (Figure 1c,

$83 \mathrm{~S} 1 \mathrm{~b}, \mathrm{~S} 1 \mathrm{e}, \mathrm{S} 1 \mathrm{f})$. The temporal decrease in the mesendoderm fraction occurred despite the cells'

84 continued ability to respond to BMP and Activin signals throughout this period (Figure S1g, S1h).

85 These findings suggested that the probability of cells transitioning to the mesendodermal fate in

86 response to signal decreases over time within the differentiating population.

\section{Dynamics of OCT4 and SOX2 predict the mesendoderm competence of individual cells}

88 We next sought to directly measure and predict the probability of individual cells adopting a

89 mesendodermal fate in response to BMP and Activin signals along the ectodermal differentiation 
90 trajectory. To do so, we first developed a measure of each live cell's position along the

91 developmental trajectory by choosing a low-dimensional coordinate - in this case, the expression

92 levels of key genes-whose dynamics accurately report on the choice of the two germ layer

93 lineages. Our recent computational work allows us to identify these key genes for a given lineage

94 decision from single cell gene expression data ${ }^{26,27}$, and we have demonstrated the accuracy of this

95 method for germ layer, neural, and hematopoietic development. For the decision between the two

96 germ layer lineages, the genes that allow us to continuously monitor the progression of fate choice

97 are the transcription factors OCT4 (POU5F1) and SOX2. Our previous work in mouse showed

98 that the protein levels of Oct4 and Sox2 reflect the transitions of pluripotent cells to the

99 mesendoderm or ectoderm fates ${ }^{26-28}$. We validated that, in humans as in mouse, both OCT4 and

100 SOX2 are symmetrically highly expressed in the pluripotent stem cell, but they are asymmetrically

101 downregulated in the two lineages. OCT4 expression is maintained in the mesendoderm while

102 SOX2 is downregulated; in contrast, ectoderm differentiation involves SOX2 maintenance and

103 OCT4 downregulation. Both TFs are also functionally important for these state transitions: OCT4

104 downregulation is necessary for neurectoderm induction ${ }^{28,29}$, while SOX2 downregulation is

105 required for mesendoderm fate selection ${ }^{28}$. Furthermore, direct conversion to a neural fate silences

$106 \mathrm{OCT}^{28}$, underscoring the fundamental nature of this reaction coordinate to the fate decision in

107 question.

108 To monitor developmental trajectories in real time, we employed our validated hESC line in which

109 one allele each of OCT4 and SOX2 had been replaced with OCT4:RFP and SOX2:YFP,

110 respectively, at the endogenous $\operatorname{locus}^{30}$ (Figure S2a-c). Using flow cytometry, we followed the

111 developmental trajectories of hESCs to ectodermal progenitors as they downregulated OCT4 over 
112 the course of six days. When BMP4 and Activin A signals were added at an intermediate stage of

113 differentiation (Figure 2a), we could visualize a bifurcation of developmental trajectories: the

114 mesendoderm-competent cells adopted $\mathrm{OCT}^{+}{ }^{+} \mathrm{SOX} 2^{-}$mesendoderm-derived fates, while the cells

115 that were not mesendoderm-competent proceeded towards OCT4- $\mathrm{SOX}^{+}$ectoderm-derived fates.

116 We next sought to measure the probability of an individual cell adopting a mesendoderm-derived

117 fate given the cell's location along the ectodermal trajectory. To do so, we performed a time lapse

118 experiment using the OCT4:RFP SOX2:YFP hESC line (Figure 2b). To monitor the cells

119 throughout this process, we developed a custom live-cell microscopy setup that was capable of

120 imaging cells on the flexible membrane every 15 minutes for over five days (Figure S2d). Based

121 on the timing of mesendoderm competence loss in our flow cytometry experiments, we first

122 differentiated the pluripotent stem cell population in this apparatus for 2.25 days in ectodermal

123 differentiation conditions to obtain a heterogeneous population in which some cells had already

124 lost mesendoderm competence and some had not. We then added BMP4 and Activin A signals for

12525 hours, prompting mesendoderm-competent cells to adopt mesendoderm fates and non-

126 mesendoderm-competent cells to adopt ectodermal fates.

127 Using our time lapse data, we demonstrated that each cell's location along its developmental 128 trajectory as measured by OCT4:RFP and SOX2:YFP levels was predictive of the cell's 129 mesendoderm competence, and that this measure allowed such prediction days before the 130 expression of classical master regulators. We tracked individual cells from pluripotency through 131 ectoderm-directed differentiation and subsequent BMP4 + Activin A signal (Figure 2b). 132 Pluripotent cells were tightly localized in OCT4:RFP/SOX2:YFP space but downregulated 

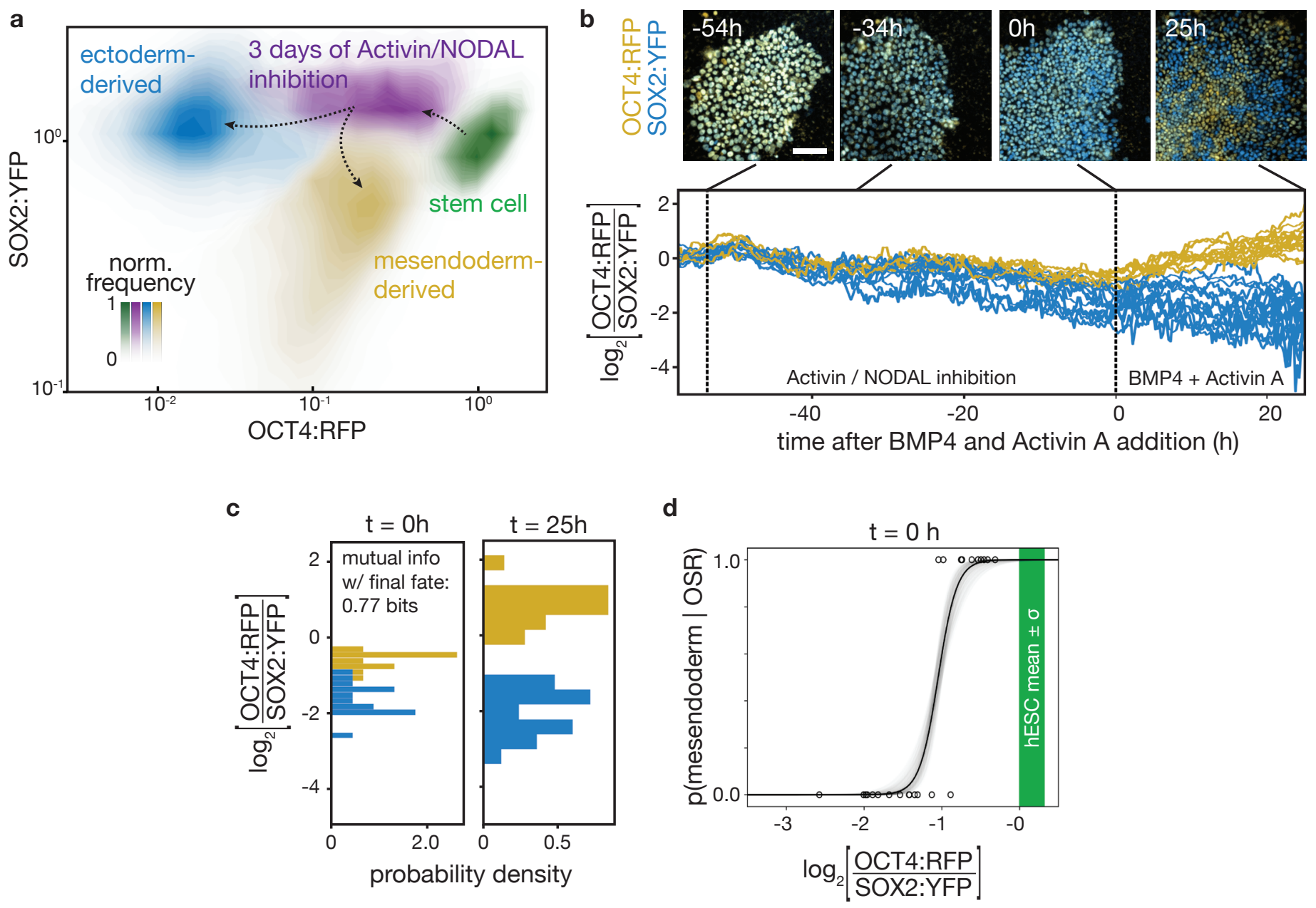

Figure 2 
Figure 2: Dynamics of OCT4 and SOX2 accurately predict mesendoderm competence.

a, Contour plots of flow cytometry data showing levels of OCT4:RFP and SOX2:YFP normalized to the mean level in the hESC population. hESCs (green) downregulate OCT4:RFP after 3 days of ectodermal differentiation (purple). After a subsequent two days of BMP4 + Activin A stimulation, the cells in this purple population bifurcate: the competent fraction chooses a mesendodermal fate (yellow), while the remaining continue to become ectoderm (blue). b, Top, snapshots from a timelapse microscopy experiment of a field of hESCs showing endogenous OCT4:RFP (yellow) and SOX2:YFP (blue). Cells started in pluripotency conditions; at $\mathrm{t}=-54 \mathrm{~h}$, ectodermal differentiation began; at $\mathrm{t}=0 \mathrm{~h}, \mathrm{BMP} 4+$ Activin A stimulation began; at $\mathrm{t}=25 \mathrm{~h}$, experiment ended. Scale bar $=100$ $\mu \mathrm{m}$. Bottom, plot of the log ratio of the OCT4:RFP to SOX2:YFP signal (OSR) in individual cells through the time course above. Time traces of cells adopting a mesendodermal (ectodermal) fate at the end of the time course are shown in yellow (blue). c, Histogram of the log OSR from (b) shown at the time of signal addition $(\mathrm{t}=0 \mathrm{~h})$ and at the end of the experiment $(\mathrm{t}=25 \mathrm{~h})$. Histograms corresponding to cells adopting an eventual mesendodermal (ectodermal) fate at the end of the time course are in yellow (blue). The mutual information between the OSR at the moment of signal addition and the final fate choice is 0.77 bits. $\mathbf{d}$, Plot of the probability of a single cell adopting a mesendoderm-derived fate given OSR, $p$ (mesendoderm | OSR), calculated from time course in (b), mean black, 1 std in grey (see Methods). Green bar: mean value $+/-$ std of the ratio in pluripotent stem cells. 
133 OCT4:RFP at different rates upon ectoderm differentiation. Importantly, we found that, rather than

134 the levels of the individual proteins, the OCT4:RFP to SOX2:YFP fluorescence ratio at the

135 moment of BMP4 and Activin A signal addition was predictive of the ultimate fate of the cells

136 with high accuracy (Figure 2c). Each cell carried 0.77 bits of mutual information (the maximum

137 possible value being 1) about its mesendoderm competence state in its ratio of OCT4:RFP to

138 SOX2:YFP at the moment of signal addition. Cells with a high ratio of OCT4:RFP to SOX2:YFP

139 were able to become mesendoderm in response to the BMP4 and Activin A signal, while cells with

140 a low ratio of OCT4:RFP to SOX2:YFP were not.

141 We then computed the probability of a cell adopting a mesendodermal fate given its OCT4:RFP

142 to SOX2:YFP ratio (OSR) after normalizing OCT4:RFP and SOX2:YFP intensities to the mean

143 levels measured in hESCs in pluripotency conditions (Figure 2d). The probability of adopting a

144 mesendodermal fate given OSR, $p$ (mesendoderm|OSR), had a sharp transition from 1 to 0 ,

145 indicating that there was a defined point along the developmental trajectory at which cells lose

146 their ability to become mesendoderm even when exposed to the relevant signals. On the

147 Waddington landscape, the OSR corresponds to a cell's position along the ectoderm trajectory,

148 and the point at which $p$ (mesendoderm|OSR $)$ transitions from 1 to 0 corresponds to the location

149 of the barrier that arises between the two fates.

150 Prospective isolation of cell populations based on mesendoderm competence

151 Having computed $p$ (mesendoderm|OSR) for single cells in our time lapse, we sought to predict

152 the mesendoderm competence of cells in a heterogeneous differentiating population. Since cells in

153 a population move along the developmental trajectory at differing rates, at any given time, $t$, the 
154 cells have a distribution of OSRs, $p(\mathrm{OSR} \mid t)$. In the Waddington landscape, this corresponds to

155 the distribution of cell locations along the ectoderm trajectory at that given time. The fraction of

156 the overall cell population that adopts a mesendoderm fate after BMP4 and Activin stimulation

157 should be determined by the fraction of the cells that have not yet reached the point along the

158 ectodermal trajectory where the barrier to mesendoderm arises. Thus, the total fraction of cells that

159 become mesendoderm can be calculated by multiplying the fraction of cells with a given OSR by

160 the probability that cells with that ratio will become mesendoderm, then summing over all OSRs

161 (Figure 3a):

$162 \quad$ Equation 1: $p($ mesendoderm $\mid \mathrm{t})=\sum_{\text {OSR }} p($ mesendoderm $\mid \mathrm{OSR}) \times p(\mathrm{OSR} \mid \mathrm{t})$

163 We next reasoned that we should be able to use our ability to predict mesendoderm competence to

164 prospectively isolate competent from non-competent cells from a single differentiating population.

165 To this end, we measured $p(\mathrm{OSR} \mid t)$ of a population of differentiating hESCs using flow

166 cytometry. Our analysis suggested that the cells in the high OSR region where our computed

$167 p($ mesendoderm $\mid \mathrm{OSR}) \approx 1$ would be competent to differentiate into mesendoderm, while those

168 in the low OSR region where $p($ mesendoderm $\mid$ OSR $) \approx 0$ would not (Figure $3 b$ ). We will

169 hereafter refer to these populations as "pre-competence-loss" and "post-competence-loss,"

170 respectively. To validate our predictions, we sorted cells based on their OSR using fluorescence-

171 activated cell sorting (FACS) from a population that had been subjected to 3 days of ectodermal-

172 directed differentiation (Figure 3c). We then added BMP4 and Activin A to the sorted populations

173 to compare our predicted mesendodermal competence with the observed fate choice of these cells. 
a

$$
\underset{\text { mesendo }}{\text { fraction }}=\sum_{\text {OSR }}
$$

p(mesendoderm | OSR)

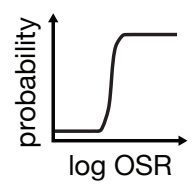

c

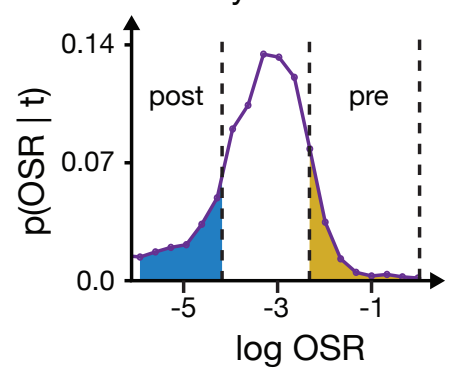

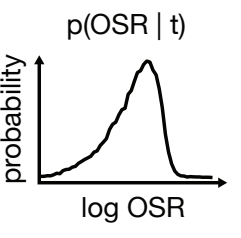

d

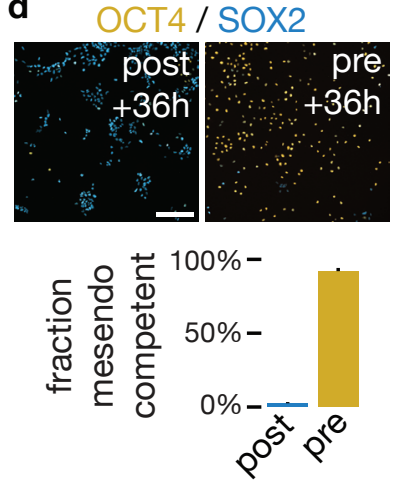

Figure 3 b
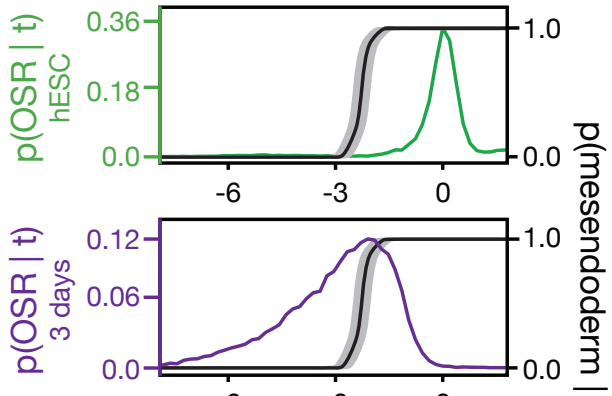

$\begin{array}{lll}-6 & -3 & 0\end{array}$

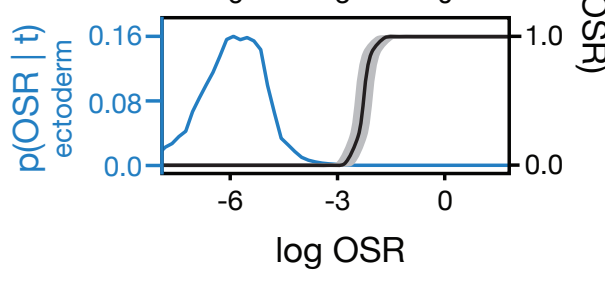




\section{Figure 3: Prospective isolation of cell populations based on mesendoderm competence.}

a, The predicted fraction of cells that adopt a mesendodermal fate upon BMP4 and Activin stimulation at a given time $t, p($ mesendoderm $\mid t$ ), equals the sum over OSR of the probability of adopting a mesendodermal fate given OSR, $\mathrm{p}$ (mesendoderm $\mid$ OSR), times the probability distribution of OSR in the population at that given time, $p(O S R \mid t)$. b, Plot of $p(O S R \mid t)$ as a function of OSR for the populations in (a) shown using the same color: pluripotent stem cells (green), 3 days of ectodermal differentiation (purple), ectodermal population (blue). $\mathrm{p}$ (mesendoderm | OSR) (black curve) inferred based on the final mesendoderm fraction from (a). $\mathrm{p}(\mathrm{OSR} \mid \mathrm{t})$ moves leftward as cells differentiate. The cells in the purple population corresponding to the region with $\mathrm{p}($ mesendoderm $\mid \mathrm{OSR})=1$ are predicted to be mesendoderm competent while the cells where $\mathrm{p}($ mesendoderm $\mid \mathrm{OSR})=0$ are predicted not to be. $\mathbf{c}$, Histogram showing $\mathrm{p}(\mathrm{OSR} \mid \mathrm{t})$ for a cell population at $t=3$ days of Activin/NODAL inhibition. Overlaid on the histogram are the FACS gates based on (f) used to sort subpopulation "post," shown in blue, predicted to have lost mesendoderm competence; and subpopulation "pre," shown in yellow, predicted to retain mesendoderm competence. d, Top, images of pre- and post-competence-loss subpopulations after cell sorting by FACS followed by 40h of BMP4 + Activin A stimulation and immunostaining for OCT4 (yellow) and SOX2 (blue). Scale bar $=300 \mu \mathrm{m}$. Bottom, fraction of cells in the pre and post sorted population adopting mesendodermal fate. Error bars: std $(n=3)$. The sorted populations essentially uniformly adopt the predicted fate. 
174 As predicted, we obtained essentially pure populations of OCT4- $\mathrm{SOX}^{+}$ectoderm-derived fates

175 from the post-competence-loss population and $\mathrm{OCT}^{+} \mathrm{SOX}^{-}$mesendoderm-derived fates from

176 the pre-competence-loss population (Figure 3d). Thus, we were indeed able to prospectively

177 isolate individual cells at points before and after loss of mesendodermal competence.

178 Having achieved our first goal of predicting mesendoderm competence, we turned to our second

179 goal: to change this competence by modulating the Waddington landscape. We hypothesized that

180 we could change the fraction of mesendoderm competent cells in one of two ways: (1) by altering

181 the position of cells along the ectoderm-directed developmental trajectory, $p(\mathrm{OSR} \mid t)$, so that more

182 or fewer cells would be past the point of competence loss at the time of BMP4 + Activin A signal

183 addition, or (2) by modulating mesendoderm competence along the trajectory,

$184 p$ (mesendoderm|OSR), such that competence loss (the sharp drop-off in probability from 1 to 0 )

185 happens earlier or later along the trajectory. We further hypothesized that important factors

186 controlling these two aspects would be DNA-binding factors whose expression patterns or access

187 to binding sites changed along the developmental trajectory. Thus, to identify candidate factors

188 that control these two probabilities, we characterized pre- and post-competence-loss populations

189 using RNA sequencing (RNA-seq) and Assay for Transposase-Accessible Chromatin using

190 sequencing (ATAC-seq).

\section{Key TF families are remodeled upon loss of mesendoderm competence}

192 For our RNA-seq and ATAC-seq experiments, we isolated populations of pre- and post193 competence cells using FACS from a single heterogeneous population of stem cells that had been

194 subjected to 3 days of ectodermal differentiation. As a control, we reserved a small fraction of 
195 sorted cells from each sample that we then treated with BMP4 and Activin A to confirm the

196 competence of that sorted population. We also included a third, mesendoderm-derived population

197 produced by subjecting pluripotent stem cells to BMP4 + Activin A for 40 hours, which allowed

198 us to distinguish lineage-specific changes in expression and chromatin accessibility from changes

199 that are shared by cells entering either lineage (Figure S3a). These populations displayed

200 significant and concerted changes in both their RNA-seq and ATAC-seq signatures upon the loss

201 of mesendoderm competence (Figure 4a).

202 Differential analysis of our RNA-seq data between the pre- and post-competence-loss populations

203 using mesendoderm as an outgroup (see methods) showed that 544 genes were upregulated

204 specifically in the post-competence-loss cells, 23 of which were TFs (Figure 4b, S3b). We also

205 found 673 genes (32 TFs) that were specifically downregulated. In particular, we observed the

206 differential expression of TFs such as SOX9, HESX1, LHX2, FOXB2, TFAP2A, TFAP2C,

207 PKNOX2, ZEB1, ZEB2, and GBX2, along with the expected expression pattern of OCT4 (Figure

208 S3c). Consistent with our earlier observation (Figure S1g-h), the differentially expressed genes did

209 not include signaling pathway components (Figure S3d). Our data further showed that competence

210 loss occurred prior to the expression of master regulators such as PAX6 and SOX1 (Figure S3c).

211 The expression pattern of all TFs is plotted in Figure S3e.

212 We validated our findings with complementary analysis of GO term enrichment (Figure S3f) and

213 ChIP-seq target enrichment (Figure S3g). Again, we observed that genes that are specifically

214 downregulated upon competence loss are most enriched for putative targets of factors previously

215 implicated in pluripotency and germ layer selection, such as SOX2, ZEB1, SMAD4, TCF3, and 
a

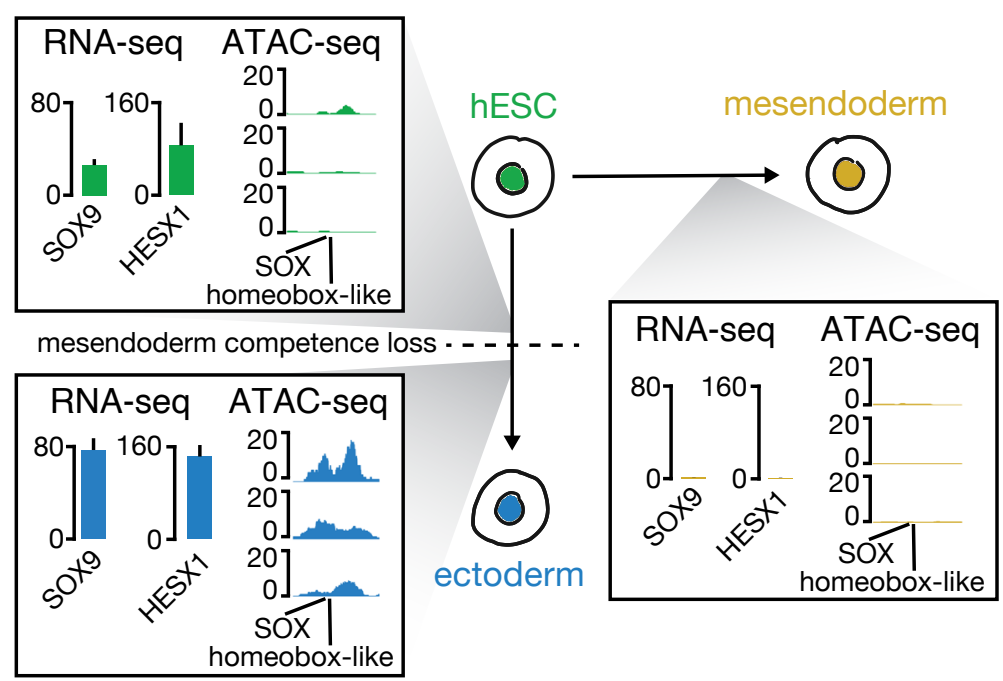

b

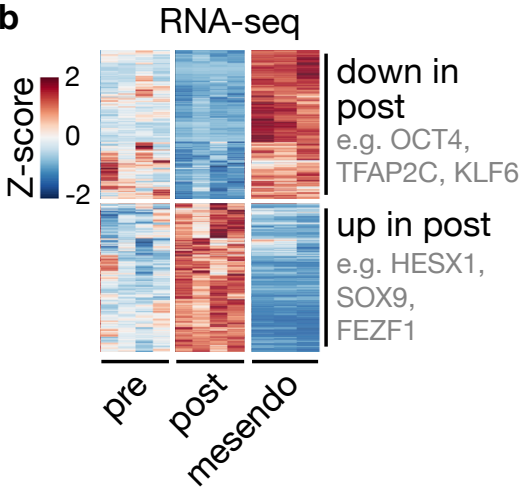

d

TF that is c ATAC-seq
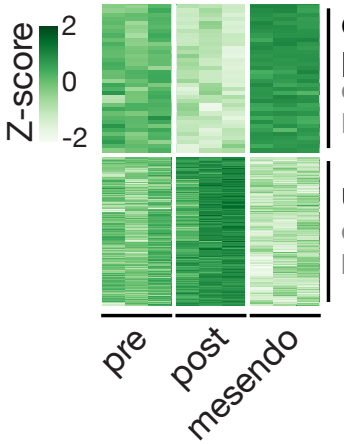

down in

post

e.g. FOX,

POU, ZEB

up in post

e.g. SOX,

homeobox diff. expressed in RNA-seq

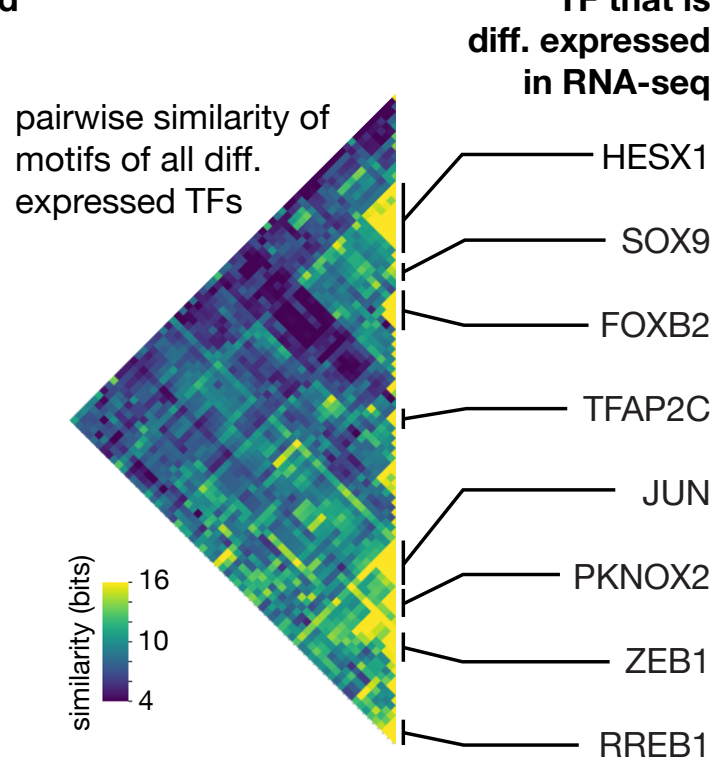

TF family with sig. in both RNA-seq and ATAC-seq

TAATTA-binding

SOX

FOX

AP-2

AP-1

PKNOX/MEIS

ZEB

RREB motif enriched in ATAC-seq analysis

${ }_{0}^{2}=$ IAAIIA

${ }_{0}^{2}$ 드프 즈

${ }_{0}^{2} \mathrm{~A} A \mathrm{ACAA}$

i. $\triangle C C_{x_{0} S S C C}$

2IATGACTCAI

$\left.{ }_{2}^{2}\right)_{x}$ TGACAG $=$

${ }_{0}^{2} \mathrm{CACCTG}$

${ }_{0}^{2} G_{A} \mathrm{CC} C \mathrm{C} \subset \mathrm{C} C \mathrm{~A}_{C}$

Figure 4 


\section{Figure 4: Key TF families show concordant changes in expression and motif accessibility} upon mesendoderm competence loss, suggesting perturbation candidates

a, Illustration showing the three assayed populations: pre- and post-competence loss (Left) and mesendoderm (Right). Inset boxes illustrate, with one example, combined analysis of gene expression and chromatin accessibility data from the three populations. Within each inset: Left, gene expression of SOX9 and HESX1 measured in transcripts per million, error bars: sd ( $\mathrm{n}=3$ ). Right, ATAC-seq read depth for three biological replicates at a genomic locus that contains SOX and TAATTA homeobox-like motifs, to which SOX9 and HESX1, respectively, are known to bind. SOX9 and HESX1 are upregulated in the post-committed population along with increased chromatin access to their binding site. b, Shown are all genes with significant lineage-specific differential expression level changes (Heat map with Z-scores) between the pre-competence-loss and post-competence loss populations $(n=4)$ as well as mesendoderm-derived outgroup $(n=3$ biological replicates). Key TFs downregulated (upregulated) upon loss of mesendoderm competence include OCT4, TFAP2C, and KLF6 (HESX1, SOX9, and FEZF1). c, Heatmap showing row-normalized ATAC-seq read depth in all 250 bp peaks with a significant change in read depth between competent and non-competent populations $(n=3)$. Like gene expression, these regions display clear, commitment and lineage-specific accessibility changes. d, Left, heatmap showing an information-based measure of similarity (see Methods) between the known DNA binding motifs of all pairs of differentially expressed TFs. Each row/column corresponds to the motif of one TF, and the matrix is arranged using hierarchical clustering. Only one half of the symmetric matrix is shown. TFs with similar preferences for DNA primary sequence cluster together, and notable families are labeled with the name of one cluster member in the column at Center Left. Center Right, the name of the TF family whose signatures are seen in both the RNAseq analysis and ATAC-seq analysis. Right, the corresponding motif identified as significant in the ATAC-seq analysis for each labeled TF family. These key TF families create concordant signatures in gene expression and chromatin accessibility data during mesendoderm competence loss, and are good candidates for perturbing mesendoderm competence. 
$216 \mathrm{KLF} 4$; in contrast, genes that are specifically upregulated upon mesendoderm competence loss are

217 most enriched for putative targets of Polycomb repressive complex 2 component SUZ12, which is

218 known to repress ectoderm target genes until their expression is appropriate.

219 From our ATAC-seq analysis, we observed DNA accessibility peaks that showed reproducible,

220 clear changes between groups, alongside many peaks that were present in all samples (Figure S4a).

221 Accessibility, as assayed by read depth, showed clear peaks at transcription start sites (Figure S4b).

222 Differential analysis of our ATAC-seq data between the pre- and post-competence-loss

223 populations using mesendoderm as an outgroup (see Methods) showed thousands of regions that

224 change accessibility between pre- and post-competence-loss populations. We found 2071 regions

225 that were more accessible after competence loss, and 233 that were less accessible (FDR $<0.05$;

226 Figure 4c). Most of these differentially accessible regions were located in distal intergenic or

227 intronic regions (Figure S4c).

228 We next confirmed that we could reproduce expected patterns in our ATAC-seq data. Using the 229 software GREAT ${ }^{31}$, we found that genes near loci with increased accessibility in the post230 competence-loss samples were most significantly enriched for orthologs of mouse genes expressed

231 in the Theiler Stage 11 neurectoderm (Figure S4d), increasing our confidence that we were indeed 232 sampling the transition we hoped to capture. We further noted that we could recover previously 233 hypothesized motif signals from our data. For example, our ATAC-seq peaks that changed 234 significantly and which also contained a compressed OCT4/SOX17 motif were almost exclusively 235 open in the mesendoderm-derived outgroup (Figure S4e), consistent with what would be predicted 236 from previous work ${ }^{32}$; in contrast, such regions that contained the classical OCT4/SOX2 motif 
237 were generally closed in the mesendoderm-derived cells. Interestingly, we did not observe

238 significant changes in accessibility at any of the ENCODE-annotated candidate regulatory

239 elements of pluripotency genes such as OCT4, SOX2, NANOG, KLF4, and MYC (Figure S4f).

240 To find TFs that potentially bind to the differentially accessible regions, we searched for sequence

241 motifs that were enriched at these loci. We found more than 20 such motifs, many of which

242 matched the known DNA-binding motifs of the differentially expressed TF families we had

243 identified, including motifs that were similar to those bound by SOX, forkhead box (FOX), AP-2,

244 AP-1, TAATTA-binding homeobox-like, PKNOX/MEIS, Zinc Finger E-Box Binding Homeobox

245 (ZEB), and POU family TFs (Figure S4g), the latter of which includes OCT4 as a member. As a

246 complementary analysis, we determined which known sequence motifs could best explain the

247 changes in chromatin accessibility that we observed across the point of competence loss (Figure 248 S4h).

249 We clustered the known binding motifs of the differentially expressed TFs by calculating a 250 measure of similarity between each pair of motifs, and we found that many such TFs shared similar 251 binding motifs (Figure 4d). These clusters correspond to major TF families - including the FOX, 252 SOX, AP-2, PKNOX/MEIS, ZEB, and homeobox-like TAATTA-binding TF families-and 253 multiple members of each family are differentially expressed. Each of these major TF family DNA 254 binding motifs was also enriched in the ATAC-seq analysis, indicating that the expression changes 255 of these TFs have clear signatures in the chromatin accessibility data. Taken together, the gene 256 expression and chromatin accessibility data revealed that a small, core set of TFs from key families 257 are remodeled upon loss of mesendodermal competence (Figure S5a). 
Key TFs independently modulate barrier between fates and location along developmental

We hypothesized that perturbation of these factors could modulate the position of cells along the

ectoderm-directed developmental trajectory, $p(\mathrm{OSR} \mid t)$, or the cell's mesendoderm competence

262 along the trajectory, $p$ (mesendoderm|OSR). To test these predictions, we selected 36 genes,

263 composed largely of the differentially expressed TFs in our core network plus their paralogs

264 (Figure S5b and S5c; see Methods), for overexpression studies. We seeded two parallel samples

265 of pluripotent stem cell populations for each candidate gene. Using a lentiviral delivery system,

266 we transduced cells in both samples with a payload of a single gene of interest separated from the

267 C-terminal end of a mCerulean cyan fluorescent protein (CFP) by a P2A self-cleaving peptide

268 sequence, all under the control of an EF-1 $\alpha$ promoter (Figure S6a). Viral transduction was initiated

269 at the onset of ectoderm-directed differentiation (Figure S6b). After three days of differentiation,

270 we analyzed one sample by flow cytometry to measure $p(\mathrm{OSR} \mid t)$. We switched the other sample

271 to media containing BMP4 + Activin A for 42 hours and assayed the final fate fractions by flow

272 cytometry. We confirmed that our non-transduced controls had produced about 50\% OCT4:RFP

273 SOX2:YFP- mesendoderm-derived cells and 50\% OCT4:RFP- SOX2:YFP ${ }^{+}$ectoderm-derived

274 cells. The transduced cells, in contrast, expressed CFP (Figure S6c) and displayed varying final

275 lineage proportions. The co-expressed CFP marker allowed us to monitor transduction efficiency

276 at the single-cell level. We titrated viral concentration to achieve $<50 \%$ transduction so that each

277 sample also contained many non-transduced ( $\left.\mathrm{CFP}^{-}\right)$cells to serve as an internal control population.

278 In principle, a change in the fraction of lentiviral-perturbed cells that form mesendoderm-derived

279 cell types after BMP4 and Activin A treatment could result from either of the hypothesized effects: 
280 first, the perturbation could facilitate or impede the initial ectoderm-directed differentiation, 281 thereby changing $p(\mathrm{OSR} \mid t)$; second, the perturbation could change $p($ mesendoderm $\mid \mathrm{OSR})$, the 282 probability of transitioning to mesendoderm given the location along the developmental trajectory. 283 For each candidate TF, we computed $p($ mesendoderm|OSR) based on the $p(\mathrm{OSR} \mid t)$ and the 284 observed final fraction of mesendoderm of lentiviral-perturbed cells (Equation 1).

285 For certain candidate TFs, their overexpression principally affected mesendoderm competence by 286 shifting the barrier between fates, captured by $p$ (mesendoderm|OSR). For example, 287 overexpression of JUNB, POU2F3, or FOXB2 increased mesendoderm competence by shifting $288 p($ mesendoderm|OSR) (Figure 5a). We concluded that these candidates tuned mesendoderm 289 competence by moving the barrier between fates on the developmental landscape.

290 In contrast, other candidates affected progression along the developmental trajectory, captured by $291 p(\mathrm{OSR} \mid t)$. For example, overexpression of SOX9 facilitated movement along the developmental 292 trajectory, thereby shifting the distribution of $p(\mathrm{OSR} \mid t)$ towards the ectoderm fates (Figure S6d). 293 In contrast, overexpression of TFAP2C hindered movement along the developmental trajectory, 294 thereby shifting $p(\mathrm{OSR} \mid t)$ towards the pluripotent state. We concluded that these candidates tuned 295 fate competence by changing cellular position along the developmental trajectory.

296 Notably, our candidates impacted competence by tuning $p($ mesendoderm $\mid \mathrm{OSR})$ and $p(\mathrm{OSR} \mid t)$ 297 independently of one another (Figure 5b, S6e). For example, while overexpression of FEZF1 and 298 TFAP2A both shifted $p(\mathrm{OSR} \mid t)$ towards the pluripotent state, they shifted $p($ mesendoderm $\mid \mathrm{OSR})$ 299 in opposite directions. The outcomes seen upon overexpression of FEZF1, TFAP2A, OTX2 and 
a

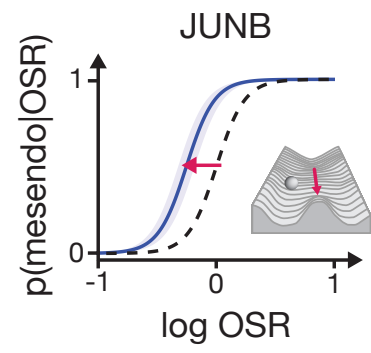

b

FEZF1

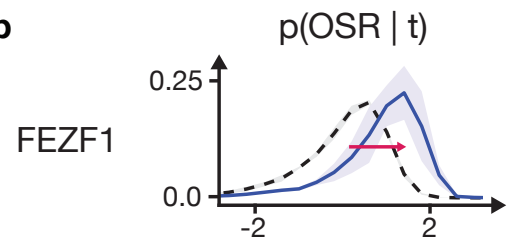

TFAP2A

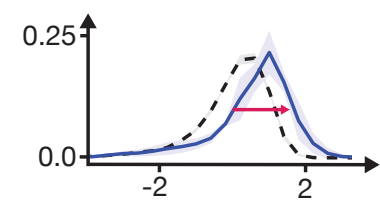

OTX2

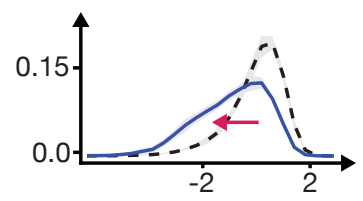

GRHL1

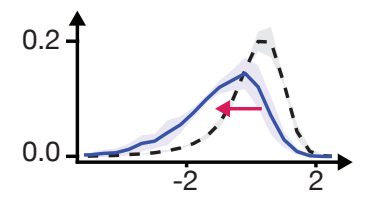

$\log$ OSR

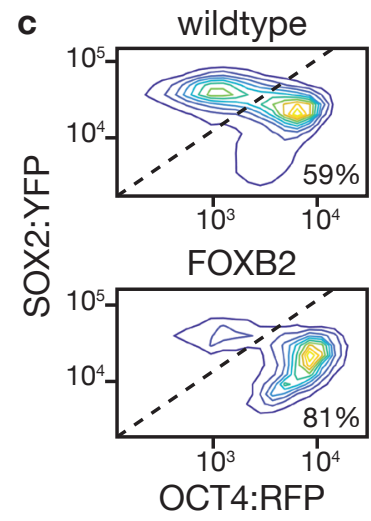

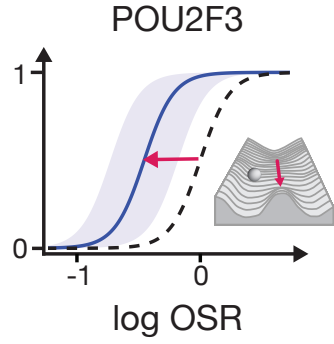

$\mathrm{p}$ (mesendoderm | OSR)
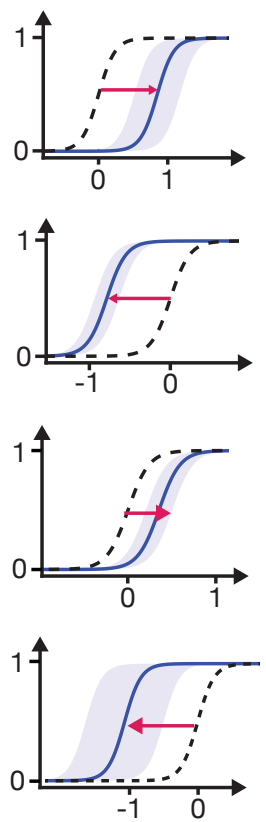

$\log$ OSR
FOXB2

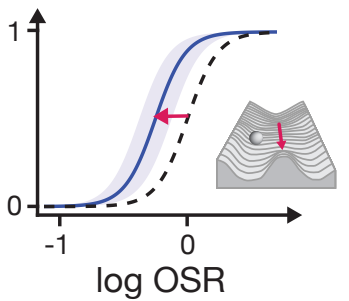

developmental landscape
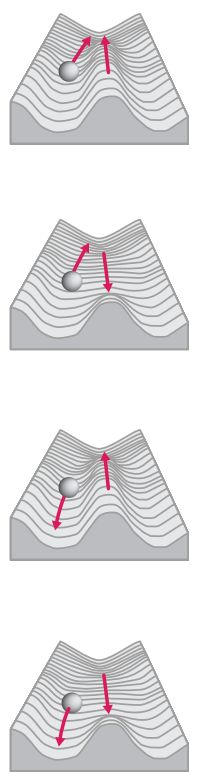

e

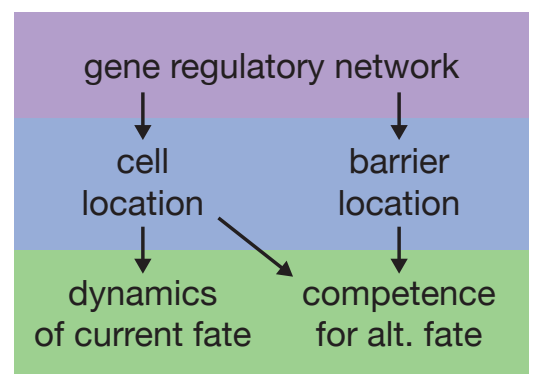

gene expression

developmental landscape

phenotype 
Figure 5: Perturbation of TFs candidates from RNA-seq and ATAC-seq analysis can independently tune progression along the developmental trajectory and inter-fate barrier.

a, Over expression of three candidates, JUNB, POU2F3, and FOXB2, shifted p(mesendoderm | OSR) to keep cells mesendoderm competent for longer along the ectodermal differentiation trajectory. Black dotted: wildtype; blue: transduced cells; shaded: std ( $n=3$, FOXB2 $n=4)$. Inset, schematic depicting the effect of overexpression on the Waddington landscape. $\mathbf{b}$, Over expression of FEZF1, TFAP2A, OTX2, and GRHL1 (top to bottom) altered both $\mathrm{p}(\mathrm{OSR} \mid \mathrm{t})$ (left column) and p(mesendoderm | OSR) (middle column). Black dotted: wildtype control; blue: transduced cells, shaded: $\operatorname{std}(n=3)$. Left, $p($ OSR $\mid t)$. Center, $p($ mesendoderm | OSR). Right, schematic as in (a). Overexpression of candidates can tune $\mathrm{p}(\mathrm{OSR} \mid \mathrm{t})$ and $\mathrm{p}$ (mesendoderm $\mid$ OSR) independently. c, Top, FACS density plot (OCT4 vs SOX2) of wild type cells (un-transduced CFP-) showing two peaks corresponding to ectodermal lineage above diagonal (41\% of cells) and mesendodermal lineage, below diagonal (59\% of cells). Bottom, transduced cells (CFP+) from the same population with CFP:P2A:FOXB2 cassette show $81 \%$ of cells adopting mesendodermal fate. Overexpression of FOXB2 increases the fraction of cells that adopt a mesendodermal fate. d, Fraction of cells adopting ectodermal fate (PAX6+) under ectodermal differentiation conditions. Cells with CFP:P2A:FOXB2 or the control CFP:P2A:CFP show normal ectodermal differentiation. OCT4 over expression precludes PAX6 expression and ectodermal differentiation. Error bars: std. e, The gene regulatory network governs both the progression of the cell along the developmental trajectory and shape of barrier between fates. In turn, both the cell's location on the developmental landscape and the location of the barrier determine the cell's fate in response to alternative signals. Only perturbations of the location of the barrier alters competence without altering the dynamics of movement along the original trajectory in the absence of a new signal. 
300 GRHL1 together represent all four possible combinations of directional shifts in $p(\mathrm{OSR} \mid t)$ and $301 p$ (mesendoderm|OSR). Thus, our candidates independently tuned $p(\mathrm{OSR} \mid t)$ and

$302 p$ (mesendoderm|OSR), thereby separately altering cellular location on the Waddington landscape

303 and the barrier between fates.

304 Lastly, we explored the downstream developmental consequences of mesendoderm competence 305 modulation via these two mechanisms. Overexpression of FOXB2, a candidate that increased 306 mesendoderm competence primarily via a shift in $p$ (mesendoderm|OSR), increased the fraction 307 of cells that adopted mesendoderm by $32 \pm 5 \%$ (Figure $5 \mathrm{c}$ ). Despite this change in mesendoderm 308 competence, FOXB2 overexpression did not prevent normal neurectodermal differentiation as 309 assayed by PAX6 expression in the absence of the BMP and Activin signal (Figure 5d, S6f). Thus, 310 alternative fate competence can be modulated without preventing normal lineage progression in 311 the absence of alternative-fate-inducing signals.

\section{Discussion}

313 Our finding that competence for an alternative fate can be controlled either by changing the

314 location of the cell along the developmental trajectory or moving the barrier that prevents cells

315 from crossing over to the mesendoderm valley suggests possible evolutionary and developmental

316 consequences. While moving the cell along the trajectory interferes with normal development in

317 the absence of alternative signal, moving the barrier does not (Figure 5e). These two effects

318 represent fundamentally different mechanisms for tuning competence, and both could be at play

319 in the developing embryo. During the patterning of the mammalian epiblast, for example, the

320 mesendodermal progenitors are generated along the primitive streak as it extends anteriorly from 
321 the posterior end of the epiblast. We speculate that changing the dynamics of epiblast competence

322 loss anterior to the primitive streak could be a possible mechanism for tuning the length and extent

323 of the streak. Further investigation of the role of competence modulation during mammalian

324 gastrulation could be an important element in a full description of this important process, and the

325 same mechanism could be acting to tune relative tissue sizes during any given cellular decision.

326 We expect that discovering reaction coordinates for different lineage decisions and monitoring the

327 dynamics of differentiation along these coordinates will be fruitful for dissecting many cell fate

328 decisions and state transitions. Our recent computational work has demonstrated how to find

329 candidate genes that accurately reflect any given lineage choice ${ }^{26,27}$, which greatly simplifies the

330 prospect of following this approach in another context. Indeed, several other choices and

331 competence restrictions occur immediately adjacent to the loss of mesendoderm competence in

332 the early germ layer lineage tree, such as the presumptive loss of non-neural potential during

333 neurectoderm fate determination or the loss of ectoderm competence during mesendoderm

334 differentiation. In sum, these findings reveal two independently tunable mechanisms for adopting

335 alternative fates along a developmental trajectory. Understanding how competence is tuned along

336 distinct mechanistic axes will be crucial for elucidating the dynamics of mammalian embryonic

337 patterning during development. 


\section{References}

340 1. Gilbert, S. The Developmental Mechanics of Cell Specification. in Developmental Biology

$341 \quad$ (Sinauer Associates, 2000).

342 2. Handyside, A. H. Time of commitment of inside cells isolated from preimplantation mouse 343 embryos. 45, 37-53 (1978).

344 3. Pedersen, R. A., Wu, K. \& BaLakier, H. Origin of the inner cell mass in mouse embryos: Cell 345 lineage analysis by microinjection. Dev. Biol. 117, 581-595 (1986).

346 4. Berg, D. K. et al. Trophectoderm Lineage Determination in Cattle. Dev. Cell 20, 244-255 347 (2011).

348 5. Rossant, J. \& Lis, W. T. Potential of isolated mouse inner cell masses to form trophectoderm 349 derivatives in vivo. Dev. Biol. 70, 255-261 (1979).

350 6. Rossant, J. \& Vijh, K. M. Ability of outside cells from preimplantation mouse embryos to form 351 inner cell mass derivatives. Dev. Biol. 76, 475-482 (1980).

352 7. Oron, E. \& Ivanova, N. Cell fate regulation in early mammalian development. Phys. Biol. 9, $353 \quad 045002(2012)$.

354 8. Kiecker, C., Bates, T. \& Bell, E. Molecular specification of germ layers in vertebrate embryos. $355 \quad$ Cell. Mol. Life Sci. 73, 923-947 (2016).

356 9. Tapscott, S. J. The circuitry of a master switch: Myod and the regulation of skeletal muscle 357 gene transcription. Development 132, 2685-2695 (2005).

358 10. Patthey, C. \& Gunhaga, L. Signaling pathways regulating ectodermal cell fate choices. Exp. $359 \quad$ Cell Res. 321, 11-16 (2014). 
360 11. Trompouki, E. et al. Lineage regulators direct BMP and Wnt pathways to cell-specific 361 programs during differentiation and regeneration. Cell 147, 577-589 (2011).

362 12. Mullen, A. C. et al. Master transcription factors determine cell-type-specific responses to TGF$363 \quad \beta$ signaling. Cell 147, 565-576 (2011).

364 13. Takaoka, K. \& Hamada, H. Cell fate decisions and axis determination in the early mouse 365 embryo. Development 139, 3-14 (2012).

366 14. Chng, Z., Teo, A., Pedersen, R. A. \& Vallier, L. SIP1 Mediates Cell-Fate Decisions between 367 Neuroectoderm and Mesendoderm in Human Pluripotent Stem Cells. Cell Stem Cell 6, 59-70 368 (2010).

369 15. Jang, J. et al. Primary Cilium-Autophagy-Nrf2 (PAN) Axis Activation Commits Human 370 Embryonic Stem Cells to a Neuroectoderm Fate. Cell 165, 410-420 (2016).

371 16. Sheng, G., Dos Reis, M. \& Stern, C. D. Churchill, a Zinc Finger Transcriptional Activator, 372 Regulates the Transition between Gastrulation and Neurulation. Cell 115, 603-613 (2003).

373 17. Yang, Y. et al. Pax6 Is a Human Neuroectoderm Cell Fate Determinant. Cell Stem Cell 7, 90$374 \quad 100(2010)$

375 18. Hou, P.-S. et al. LHX2 regulates the neural differentiation of human embryonic stem cells via 376 transcriptional modulation of PAX6 and CER1. Nucleic Acids Res. 41, 7753-7770 (2013).

377 19. Mizuseki, K., Kishi, M., Matsui, M., Nakanishi, S. \& Sasai, Y. Xenopus Zic-related-1 and 378 Sox-2, two factors induced by chordin, have distinct activities in the initiation of neural 379 induction. Dev. Camb. Engl. 125, 579-87 (1998).

380 20. Loh, K. M. et al. Mapping the Pairwise Choices Leading from Pluripotency to Human Bone, 381 Heart, and Other Mesoderm Cell Types. Cell 166, 451-467 (2016). 
382 21. Lupo, G. et al. Inhibition of Activin/Nodal signaling promotes specification of human 383 embryonic stem cells into neuroectoderm. Dev. Biol. 313, 107-117 (2007).

384 22. Li, L. et al. Ectodermal progenitors derived from epiblast stem cells by inhibition of Nodal 385 signaling. J. Mol. Cell Biol. 7, 455-465 (2015).

386 23. Waddington, C. H. The Strategy of the Genes. (Routledge, 1957).

387 24. Xu, X., Browning, V. L. \& Odorico, J. S. Activin, BMP and FGF pathways cooperate to 388 promote endoderm and pancreatic lineage cell differentiation from human embryonic stem 389 cells. Mech. Dev. 128, 412-427 (2011).

390 25. Li, L. et al. Location of transient ectodermal progenitor potential in mouse development. 391 Development 140, 4533-4543 (2013).

392 26. Jang, S. et al. Dynamics of embryonic stem cell differentiation inferred from single-cell 393 transcriptomics show a series of transitions through discrete cell states. Elife 6, (2017).

394 27. Furchtgott, L. A., Melton, S., Menon, V. \& Ramanathan, S. Discovering sparse transcription 395 factor codes for cell states and state transitions during development. eLife 6, 1-33 (2017).

396 28. Thomson, M. et al. Pluripotency factors in embryonic stem cells regulate differentiation into 397 germ layers. Cell 145, 875-889 (2011).

398 29. Greber, B. et al. FGF signalling inhibits neural induction in human embryonic stem cells: FGF 399 inhibits neural induction in hESCs. EMBO J. 30, 4874-4884 (2011).

400 30. Zhang, Z., Zwick, S., Loew, E., Grimley, J. S. \& Ramanathan, S. Mouse embryo geometry 401 drives formation of robust signaling gradients through receptor localization. Nat. Commun. 10, 402 (2019). 
403 31. McLean, C. Y. et al. GREAT improves functional interpretation of cis-regulatory regions. Nat.

404 Biotechnol. 28, 495-501 (2010).

405 32. Merino, F. et al. Structural basis for the SOX-dependent genomic redistribution of OCT4 in 406 stem cell differentiation. Structure 22, 1274-1286 (2014).

407 33. Etoc, F. et al. A Balance between Secreted Inhibitors and Edge Sensing Controls Gastruloid 408 Self-Organization. Dev. Cell 39, 302-315 (2016).

409 34. Buenrostro, J. D., Wu, B., Chang, H. Y. \& Greenleaf, W. J. ATAC-seq: A method for assaying 410 chromatin accessibility genome-wide. Curr. Protoc. Mol. Biol. 109, 21.29.1-21.29.9 (2015).

411 35. Benjamini, Y. \& Hochberg, Y. Controlling the False Discovery Rate: A Practical and Powerful 412 Approach to Multiple Testing. J. R. Stat. Soc. Ser. B Methodol. 57, 289-300 (1995).

413 36. McQuin, C. et al. CellProfiler 3.0: Next-generation image processing for biology. PLOS Biol. $414 \quad$ 16, e2005970 (2018).

415 37. Lambert, S. A. et al. The Human Transcription Factors. Cell 172, 650-665 (2018).

416 38. Kuleshov, M. V. et al. Enrichr: a comprehensive gene set enrichment analysis web server 2016 417 update. Nucleic Acids Res. 44, W90-W97 (2016).

418 39. Mi, H., Muruganujan, A., Ebert, D., Huang, X. \& Thomas, P. D. PANTHER version 14: more 419 genomes, a new PANTHER GO-slim and improvements in enrichment analysis tools. Nucleic $420 \quad$ Acids Res. 47, D419-D426 (2019).

421 40. Edelstein, A. D. et al. Advanced methods of microscope control using $\mu$ Manager software. $J$. 422 Biol. Methods 1, 10 (2014). 


\section{Methods}

\section{Cell lines}

426 We conducted our experiments using WA01 (H1, WiCell) human embryonic stem cells. We also

427 used an $\mathrm{H} 1$ cell line in which both OCT4 and SOX2 were tagged with fluorescent proteins as

428 previously described ${ }^{30}$. In these cells, one endogenous copy of OCT4 was replaced with

429 OCT4:tdTomato followed by an internal ribosomal entry site and a neomycin resistance gene to

430 allow for selection, and one endogenous copy of SOX2 was replaced with

431 SOX2:FLAG:Citrine:P2A:PuroR.

\section{Cell culture}

433 hESCs were cultured in 6-well tissue culture dishes treated with Matrigel (Corning) and supplied

434 with mTeSR media (STEMCELL Technologies) according to the manufacturer's specifications.

435 For routine culture, we passaged by washing with phosphate buffered saline (PBS) followed by

436 ReLeSR (Stem Cell) treatment according to the manufacturer's instructions. Cells were passaged

437 in clumps of 8-10 cells and seeded in mTeSR supplemented with the Rho-associated protein kinase

438 inhibitor $\gamma$-27632 (STEMCELL Technologies) at $10 \mu \mathrm{M}$ for the first day to improve survival. All

439 cell lines used were routinely tested for mycoplasma contamination.

440 For all experiments, we seeded cells on polyester membrane filters (Sterlitech) with $3 \mu \mathrm{m}$ pores

441 that had been treated with Matrigel. We chose this substrate to allow all cells to receive the BMP

442 and Activin/NODAL signals we added to the media. TGF- $\beta$ superfamily receptors, such as those

443 for BMP4 and Activin A, are localized basolaterally in epithelial stem cell colonies and in vivo in

444 the epiblast, so they are insulated from ligands in the apical media or luminal fluid ${ }^{30,33}$. Typical 
445 tissue culture conditions allow for only the cells on the edge of the colony to receive signals, but

446 growing cells on a membrane allows all cells in a colony access to the BMP and Activin ligands.

447 For live cell imaging, membranes were first glued to a custom $300 \mu \mathrm{m}$ thick stainless-steel washer 448 with Cytoseal 60 (Thermo Fisher), allowed to dry, sterilized with washes in $70 \%$ ethanol and with 449 UV treatment, then treated with Matrigel for cell seeding.

\section{Differentiation conditions}

451 Differentiation towards the ectoderm lineage was effected using mTeSR supplemented with 0.5 $452 \mu \mathrm{M}$ A83-01 (R\&D Systems), a small molecule inhibitor of Activin and Nodal signaling. BMP4 + 453 Activin A treatment was accomplished by treating cells with mTeSR supplemented with $3 \mathrm{ng} / \mathrm{mL}$ 454 recombinant human BMP4 protein (R\&D Systems) and $100 \mathrm{ng} / \mathrm{mL}$ recombinant human Activin

455 A protein (R\&D Systems). For neurectoderm-directed differentiation, we inhibited BMP signaling 456 with $0.5 \mu \mathrm{M}$ LDN-193189 in addition to Activin/Nodal inhibition with $0.5 \mu \mathrm{M}$ A83-01 for 6 days.

\section{Flow cytometry}

458 Cells were washed with PBS (Lonza) and removed from membranes by treatment with Accutase 459 (Innovative Cell Technologies) until the cells were dissociated, about 20 minutes. Cells were 460 analyzed on an LSRFortessa (BD Biosciences).

\section{$461 \quad$ Fluorescence activated cell sorting}

462 Accutase-dissociated cells were sorted using a BD Aria III (BD Biosciences) using a $100 \mu \mathrm{m}$ 463 nozzle. Cells were gated such that the pre-competence-loss population was taken as the cells with 464 the top 10-15\% OCT4:RFP to SOX2:YFP ratio, and the post-competence-loss population was the 
465 bottom 10-15\% OCT4:RFP to SOX2:YFP ratio. We sorted around 250,000 cells per subpopulation

466 in a typical experiment. Populations were sorted into $1.5 \mathrm{~mL}$ centrifuge tubes (Eppendorf) filled

467 with $500 \mu \mathrm{L}$ of mTeSR supplemented with $10 \mu \mathrm{M} \gamma-27632$; by the end of the sort, $\sim 800 \mu \mathrm{L}$ of

468 sheath and sorted cells had been added to each tube. After the sort had completed, we pelleted the

469 cells in a microcentrifuge at $250 \mathrm{xg}$ for 3 minutes, then resuspended in PBS.

470 For each sorted sample, about $10 \%$ of the sorted cells were reserved for competence testing to

471 confirm the pre-/post-competence-loss status of the sorted population. These cells were seeded

472 back into glass-bottom 24-well plates (Ibidi) treated with Matrigel and filled with $1 \mathrm{~mL}$ of mTeSR

473 supplemented with $\gamma$-27632 and allowed to recover for 3 hours. The media was then changed to

474 mTeSR supplemented with BMP4 and Activin A for 36 hours. Cells were fixed and stained for

475 OCT4 and SOX2 according to the protocols described under "Immunofluorescence."

\section{RNA-seq}

477 Total RNA was prepared from sorted or dissociated cells with an RNeasy Mini Kit (Qiagen)

478 according to the manufacturer's instructions. For the mesendoderm-derived outgroup samples, the

479 input to the RNA extraction kit was a cell population directly after dissociation with Accutase; for

480 FACS sorted populations, the input was sorted cells suspended in PBS. RNA integrity was

481 quantified with a TapeStation 4200 (Agilent). All RINe scores were $\geq 9.9$. Sequencing libraries

482 were prepared by the Bauer Core at Harvard University using a Kapa mRNA Hyper Prep kit with

483 Poly-A selection. Sequencing was performed on a NextSeq High output flow cell that generated

484 paired-end $38 \mathrm{bp}$ reads. We obtained $\geq 42 \mathrm{M}$ reads per sample.

\section{ATAC-seq}


ATAC-seq was performed as previously described ${ }^{34}$. Briefly, live cells were lysed and incubated with Tn5 transposase for $30 \mathrm{~min}$ at $37^{\circ} \mathrm{C}$. After DNA purification, samples were amplified for the

488 appropriate number of cycles as determined by qPCR to minimize PCR bias. Sequencing was

489 performed by the sequencing core at Massachusetts General Hospital. We obtained $\sim 100 \mathrm{M}$

490 mapped paired-end reads per sample.

\section{$491 \quad$ Plasmid construction}

492 Overexpression targets were subcloned from plasmids available through the Harvard PlasmID

493 database, where available. Other targets were cloned from complementary DNA (cDNA) libraries.

494 To prepare cDNA libraries for cloning, we differentiated human stem cells for 3 days in mTeSR $495+0.5 \mu \mathrm{M}$ A8301 and extracted RNA with RNeasy Mini Kit (Qiagen) according to the 496 manufacturer's instructions. We then performed first strand cDNA synthesis using SuperScript II 497 Reverse Transcriptase (Thermo Fisher). We amplified the relevant cDNAs using Phusion 498 polymerase (NEB) or Kapa HiFi (Kapa Biosystems). The OCT4 DNA binding domain and the 499 SOX2 DNA binding domain (OCT4DBD and SOX2DBD) were amplified from cDNA. The 500 OCT4DBD consisted of amino acids 131-296 of OCT4A (NCBI reference sequence: 501 NM_002701.5). The SOX2DBD consisted of amino acids 37-117 of SOX2 (NCBI reference 502 sequence: NM_003106.3). All cDNA-amplified clones were fully sequence confirmed by Sanger 503 sequencing (Genewiz).

504 We constructed a vector from the second-generation lentiviral transfer backbone pWPXL with an 505 EF-1 $\alpha$ promoter. pWPXL was a gift from Didier Trono (Addgene plasmid \#12257; 506 http://n2t.net/addgene:12257; RRID:Addgene_12257). We first joined sequences for fluorescent 
507 protein mCerulean (CFP) and 2A peptide P2A (a ribosomal skip sequence) with Q5 (NEB) fusion

508 PCR and added them to the pWPXL vector with Gibson Assembly Master Mix (NEB). We then

509 constructed final transfer vectors by inserting target cDNA after the P2A using Gibson assembly.

510 All constructed vectors were sequence confirmed at Gibson assembly junctions by Sanger

511 sequencing (Genewiz) prior to use. Plasmids were grown and stored in NEB Stable E. coli (NEB).

\section{Lentiviral overexpression and flow cytometry analysis}

513 To produce virus, we used jetPrime (Polyplus) according to the manufacturer's instructions to

514 transfect Lenti-X 293 T HEK cells (Takara) with lentiviral production plasmids pMD2.G and

515 psPAX2 along with our individual transfer plasmids. pMD2.G and psPAX2 were gifts from Didier

516 Trono (Addgene plasmid \#12259; http://n2t.net/addgene:12259; RRID:Addgene_12259; Addgene

517 plasmid \#12260; http://n2t.net/addgene:12260; RRID:Addgene_12260) We collected viral media

518 at 24 and 48 hours and concentrated using Lenti-X Concentrator (Clontech) according to the

519 manufacturer's instructions.

520 We seeded human stem cells in mTeSR medium containing $\gamma$-27632 on Matrigel-treated

521 membrane filters as described above. We treated cells with $1 \mathrm{x}$ and $3 \mathrm{x}$ viral titer at 24 hours and 48

522 hours post-seeding, respectively, in order to obtain transduction efficiency of $\sim 10 \%$. $1 \mathrm{x}$ viral

523 treatment was performed simultaneously with the beginning of A83-01 treatment.

524 Two samples of each overexpression condition were performed in parallel. We harvested cells of 525 one sample after 3 days of treatment with A83-01 and the cells of the second sample after 3 days 526 of A83-01 followed by 42 hours of BMP4 + Activin A treatment. We analyzed each sample 527 immediately after harvest using an LSRFortessa (BD Biosciences). 
528 We analyzed differential OCT4:RFP to SOX2:YFP ratio distributions between CFP-positive and

529 CFP-negative populations of each 3-day sample by calculating the Kullback-Leibler divergence in 530 MATLAB (MathWorks). To determine differences in proportions of end fates 531 (OCT4:RFP+/SOX2:YFP- and OCT4:RFP-/SOX2:YFP+), we manually gated ectoderm and 532 mesendoderm populations using a custom MATLAB script and used identical gates for both CFP533 positive and CFP-negative populations. We performed at least 3 biological replicates for all 534 candidates that showed an initial phenotype except for SOX2, AHR, ARNT2, and GBX2, each of 535 which had two replicates. Significance compared to the CFP-overexpressing negative control was 536 determined using a two-sided $t$-test, and we controlled the FDR at $10 \%$ across the set of all 537 candidates using the method of Benjamini and Hochberg ${ }^{35}$.

\section{Immunofluorescence}

539 Cells were fixed with $4 \%$ formaldehyde for $15 \mathrm{~min}$ at room temperature. Fixed cells were treated 540 with blocking buffer (PBS $+5 \%$ normal donkey serum $+0.3 \%$ Triton $\mathrm{X}-100$ ) for $1 \mathrm{~h}$, then 541 overnight at $4^{\circ} \mathrm{C}$ with primary antibody diluted in staining buffer (PBS $+1 \%$ BSA $+0.3 \%$ Triton 542 X-100). The following primary antibodies were used: OCT4 (1:400, Cell Signaling C30A3);

543 SOX2 (1:400, Thermo Fisher BTJCE); SOX17 (1:100, R\&D Systems AF1924); phosphorylated 544 SMAD1/5/9 (1:200, Cell Signaling D5B10); phosphorylated-SMAD2 (1:200, Cell Signaling 545 E8F3R); PAX6 (1:200, DSHB AB_528427); and NANOG (1:500, R\&D Systems AF1997). The 546 PAX6 antibody was deposited to the DSHB by Kawakami, A. (DSHB Hybridoma Product PAX6). 547 After overnight incubation, samples were washed three times with PBS, then secondary antibodies 548 diluted in staining buffer were added. We used the following secondary antibodies all at a dilution 549 of 1:1000: donkey anti-rabbit Alexa 568 (Thermo Fisher), donkey anti-rat Alexa 488 (Thermo 
550 Fisher), and donkey anti-mouse Alexa 647 (Thermo Fisher). We incubated with a 300 nM DAPI

551 (Thermo Fisher) solution in PBS for 5 minutes to visualize DNA. For analysis of the resulting

552 images, we used CellProfiler $3.1 .8^{36}$ to segment well-separated nuclei for samples where

553 automated segmentation performed well (those shown in Figure 2), and we used manual

554 segmentation for more challenging images (those shown in Figures 1, S1, and S6).

\section{$555 \quad$ ATAC-seq analysis}

556 Reads were trimmed using NGmerge 0.2_dev in adapter removal mode with minimum overlap (-

557 e flag) set to 20 to remove any remaining adapter sequence. Reads were aligned to the hg38 build

558 of the human genome using bowtie2 2.2.9 using the --very-sensitive preset and with a maximum

559 fragment size of 2000, then collated with samtools 1.9. Duplicate fragments were removed with

560 picard 2.8.0. Peaks were called with MACS2 2.1.1 in callpeak -f BAMPE mode. Differentially

561 accessible peaks were identified using the Bioconductor package DiffBind 2.12.0 in R 3.6.1. Peaks

562 were annotated by genomic region type using ChIPSeeker 1.20.0.

563 For differential accessibility analysis with DiffBind and DESeq2, we used a design matrix with an

564 "sample" column, which indicated the well from which the cells had been sorted (since each pair

565 of pre- and post-competence-loss samples was derived from a single population sorted by FACS),

566 and a "competenceloss" column, which was 1 for the post- competence-loss population and 0 for

567 the pre-competence-loss and mesendoderm-derived populations. Thus, we identified regions that

568 changed specifically with competence loss while controlling for original sample identity.

569 The primary DNA sequences of differentially accessible peaks were retrieved from Ensembl and 570 examined for motifs using MEME-ChIP 5.0.3. ATAC-seq read depth was modeled as a function 
571 of known motif presence using chromVAR 1.4.1. Significant motif matches were identified with

572 FIMO 5.0.3. For the gene regulatory network, possible associations between genomic regions and

573 target genes were identified using CisMapper 5.0.5. The full list of human TFs and the motifs for

574 each TF were extracted from the list in Lambert et al. ${ }^{37}$. Mutual information between pairs of

575 motifs was calculated with a custom python script.

576 Enrichment of functions of genes near differentially accessible genomic regions was performed

577 using the web interface of GREAT ${ }^{31}$ in June 2019.

\section{$578 \quad$ RNA-seq analysis}

579 Reads were pseudoaligned using kallisto 0.45 .1 to transcripts from the human genome build hg38.

580 Differentially expressed genes were identified using DESeq2 1.24.0 on R 3.6.1. For analysis with

581 DESeq2 when comparing pre- and post-competence-loss populations, we used a design matrix

582 with an "sample" column, which indicated the well from which the cells had been sorted and a

583 "population" column, which indicated the pre- or post-competence-loss state. For comparison of

584 pre-competence-loss and mesendoderm-derived populations, our design matrix contained

585 sequencing batch and pre-competence-loss or mesendoderm-derived population identity.

586 For clustering the motifs of differentially expressed transcription factors, similarity between each

587 pair of motifs was quantified as the Kullback-Leibler divergence of the product of the two motifs

588 from a reference distribution, which was the product of two uniform motifs $(0.25$ probability for

589 each base at each position). Motif alignment was performed by calculating the aforementioned

590 divergence at each possible offset and using the maximum value obtained at any offset. This

591 calculation was performed using a custom python script. The linkage was computed using the 
592 scipy.cluster.hierarchy.linkage function from scipy 1.3.0 with the "average" clustering method and

593 the "braycurtis" distance.

594 ChIP-seq target set enrichments were calculated using the Enrichr ${ }^{38}$ web interface in July 2019.

595 GO term enrichment was calculated using the PANTHER ${ }^{39}$ web interface in July 2019.

\section{Overexpression candidate selection}

597 We selected TFs by incorporating information from both RNA-seq and ATAC-seq analyses. We

598 began with all TFs that were differentially expressed between the pre- and post-competence-loss

599 populations $(\mathrm{q}<0.05$ with DESeq2). A gene was considered to be a TFs if it was so annotated in

600 Lambert et $a{ }^{37}{ }^{37}$ We then limited this list only to those that were expressed in a lineage-specific

601 pattern and had above-background expression levels in at least one of the three populations. We

602 defined genes with a lineage-specific expression pattern as those genes that (1) were differentially

603 expressed between pre- and post-competence-loss populations and (2) either were not

604 differentially expressed from the pre-competence-loss to mesendoderm populations or were

605 differentially expressed in the opposite direction (upregulated from pre- to post- and

606 downregulated from pre- to mesendoderm, or vice versa). By these criteria, 23 TFs were

607 specifically upregulated with competence loss and 32 were specifically downregulated with

608 competence loss. We also added select paralogs of the TFs that passed our expression pattern

609 cutoffs: POU6F1, GRHL1, POU2F3, and FOXJ2, along with the OCT4 and SOX2 DNA binding

610 domains. We further restricted the list to those candidates that had a known, high-quality DNA

611 binding motif that appeared in either the DiffBind/MEME-ChIP or chromVAR analyses of our

612 data. We also added four TFs (OTX2, JUNB, ZSCAN23, and GSC) whose motifs appeared in our 
613 ATAC-seq analyses but did not pass our differential expression cutoffs. We excluded ZEB1 and

614 ZEB2 as candidates because their size precluded delivery by lentivirus. We also eliminated 10 TFs

615 for which a clone was not readily accessible to us, either from the Harvard PlasmID database,

616 Addgene, or genes that had previously been cloned from cDNA in our lab. We note that one

617 candidate that was tested before all RNA-seq analysis was complete, MBNL2, missed significance

618 cutoffs in the final analysis but is nevertheless included for completeness. After adding three

619 candidates based on the literature (NRF2, ZNF521, and ID2), we were left with 36 candidates in

620 total.

\section{Epifluorescence imaging of fixed samples}

622 Samples were imaged on a Zeiss AxioObserver Z1 inverted microscope using Zeiss 10x and 20x

623 plan apo objectives (NA 1.3) using the appropriate filter sets. Images were acquired using an Orca-

624 Flash 4.0 CMOS camera (Hamamatsu). The 43 HE DsRed/46 HE YFP/47 HE CFP/49 DAPI/50

625 Cy5 filter sets from Zeiss were used. The microscope was controlled using the ZEN software.

\section{Live cell time lapse imaging}

627 Samples were imaged on a Zeiss AxioObserver Z1 inverted microscope using a Zeiss 20x plan 628 apo objective (NA 0.8) using the appropriate filter sets and a Hamamatsu ImagEM EMCCD

629 camera. Cells were maintained in a 37 degree incubation chamber at $5 \% \mathrm{CO}_{2}$. Cells were imaged

630 every 15 minutes. Focus was maintained using a combination of Zeiss Definite Focus and, using 631 a custom script in MicroManager 2.0 beta $^{40}$, software autofocus adjustments every hour to 632 compensate for slight movement of the membrane. For maximum accuracy, cells in this time lapse 
633 were tracked manually, and the tracks were analyzed with a custom python script that performed

634 illumination profile correction and background subtraction.

\section{Confocal imaging}

636 For Figure 1 and S1, cells were imaged on a Leica inverted microscope with a Zeiss 20x objective

637 (NA 0.8) with the appropriate filter sets. Detection was performed with photomultiplier tubes (for

638 detection of Alexa 488 and Alexa 647) and a Leica HyD Photon Counter (for Alexa 568). For

639 Figure S6, cells were imaged on a Zeiss LSM 880 with Airyscan using a Zeiss 20x objective (NA

640 0.8). Detection was performed with photomultiplier tubes (Alexa 568 and Alexa 647) and a GaAsP

641 detector (CFP and Alexa 488).

\section{$642 \mathbf{p}($ mesendoderm|OSR) curve fitting and location inference}

643 For the initial $p$ (mesendoderm|OSR) curve fitting to the single cell data extracted from the time

644 lapse, we fit a two-parameter sigmoid function $\frac{1}{1+\exp (-a(x-b))}$ to the data using

645 scipy.optimize.curve_fit to minimize the squared difference between data and prediction. We used

646 the learned sigmoid shape parameter, a, for all subsequent $p($ mesendoderm $\mid \mathrm{OSR})$ inference. To

647 infer $p$ (mesendoderm|OSR) for a given population, we fit the location parameter, b, by

648 minimizing the squared difference between the observed final mesendoderm fate proportion and

649 the mesendoderm proportion predicted by $p$ (mesendoderm $\mid \mathrm{OSR})$ at varying locations, $\mathrm{b}$, given

650 the observed $p(\mathrm{OSR} \mid t)$.

\section{Ethical compliance}


652 We used hESCs in accordance with approvals by Harvard University IRB (protocol \#IRB18- 0665)

653 and Harvard University ESCRO (protocol E00065).

\section{Data availability}

655 The RNA-seq and ATAC-seq datasets generated in this study will be made available in the NCBI

656 GEO repository and the NCBI SRA by the time of publication, or in advance of that date upon

657 reasonable request. All other data are available upon reasonable request.

\section{Code availability}

659 All custom code used in this study is available from the authors upon reasonable request.

\section{Author contributions}

661 J.R.V. and S.R. conceived the project. J.R.V., R.H., S.K., and S.R. designed the experiments.

662 J.R.V., R.H., S.K., and D.V. conducted the experiments. J.R.V. and R.H. analyzed the data. J.R.V., 663 R.H., S.K., D.V., R.E.K., and S.R. wrote the manuscript.

\section{Acknowledgments}

665 We thank the staff of the Bauer Core at Harvard University for their work on the RNA sequencing

666 used in this manuscript as well as for their expertise and assistance with flow cytometry and FACS.

667 We thank the Massachusetts General Hospital sequencing core for their work on the ATAC-seq 668 data used in this manuscript, Manashree Damle for bioinformatics help, the Harvard Physics/SEAS

669 Instructional Machine Shop for making the stainless-steel washers used in the live cell imaging in 
670 this study, and the Harvard Center for Biological Imaging for the use of their equipment. We thank

671 Andrew Murray, Sean Eddy, and all of the members of the Ramanathan Lab for their helpful

672 comments. J.R.V. was funded by The Fannie and John Hertz Foundation, the National Science

673 Foundation Graduate Research Fellowship Program, and the NSF-Simons Center for

674 Mathematical and Statistical Analysis of Biology at Harvard, award \#1764269. Some confocal

675 imaging was conducted on an instrument provided by the Harvard MRSEC (DMR-1420570). This

676 work was supported in part by NIH R01GM131105-01. 
a

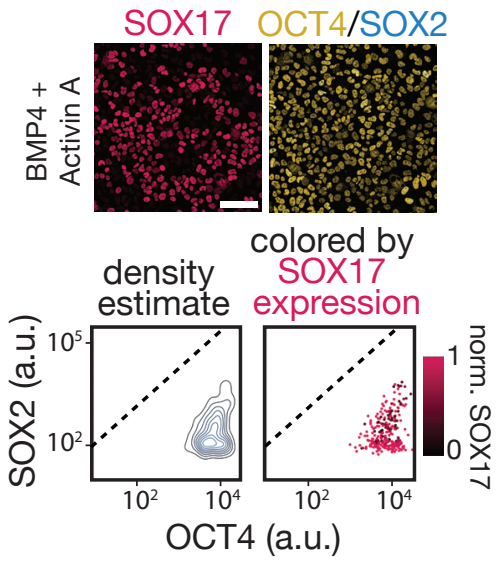

c
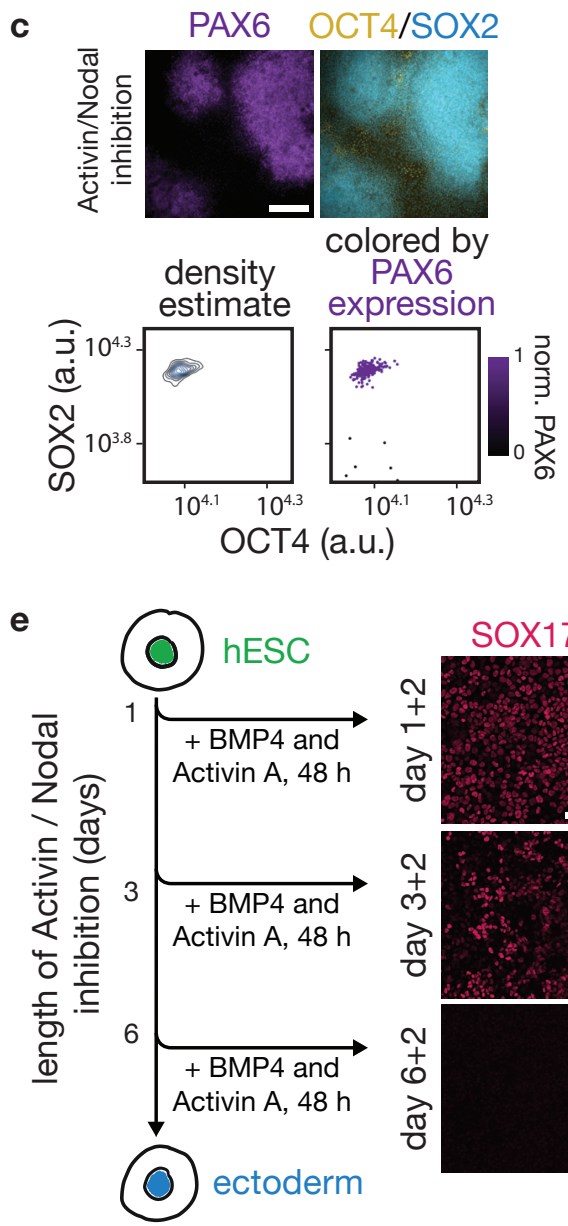

b length of Activin/Nodal inhibition before 42h BMP4 + Activin A

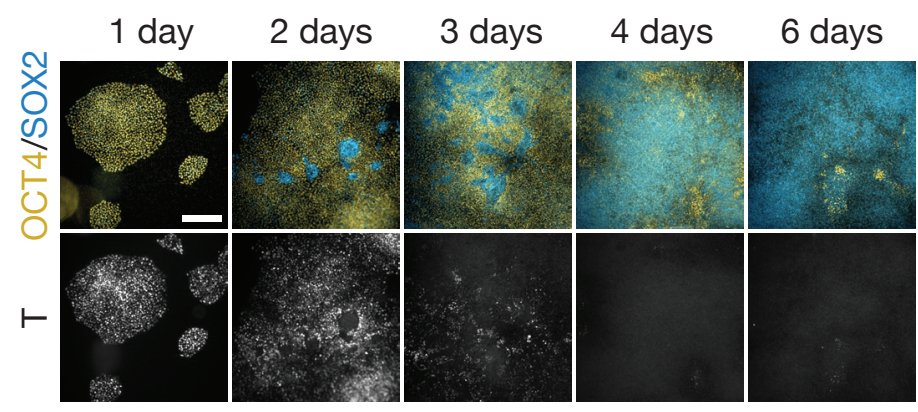

d length of Activin/Nodal inhibition with A83-01

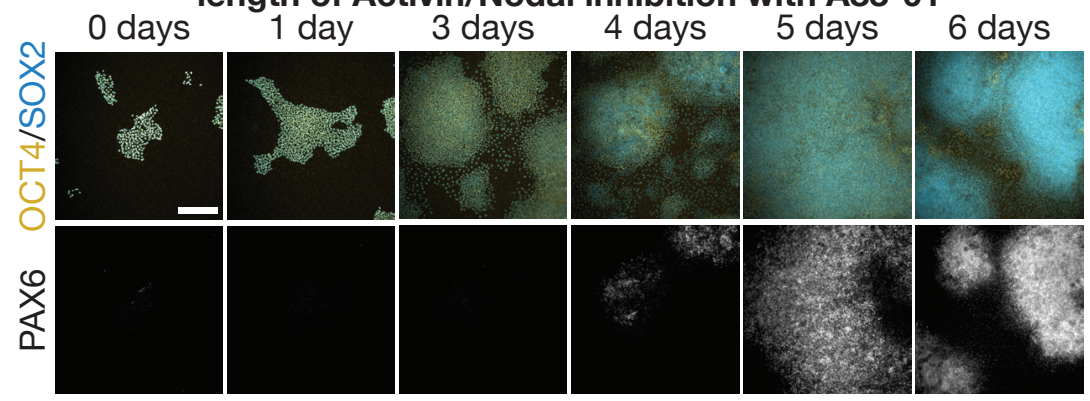

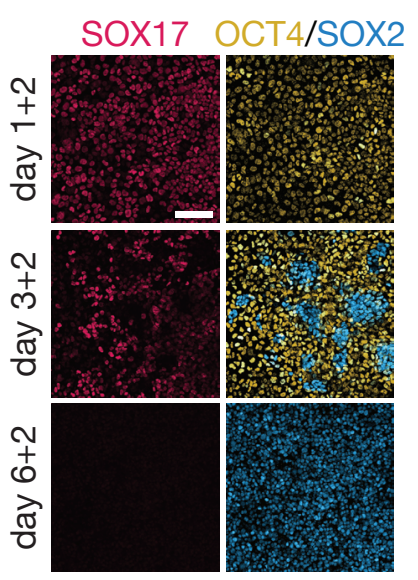

g
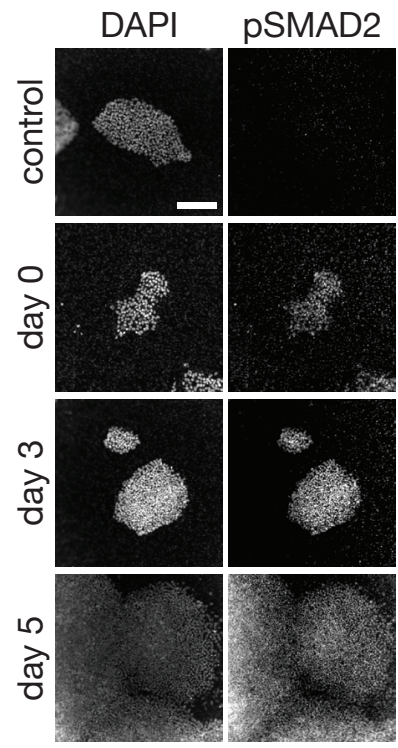

pSMAD1
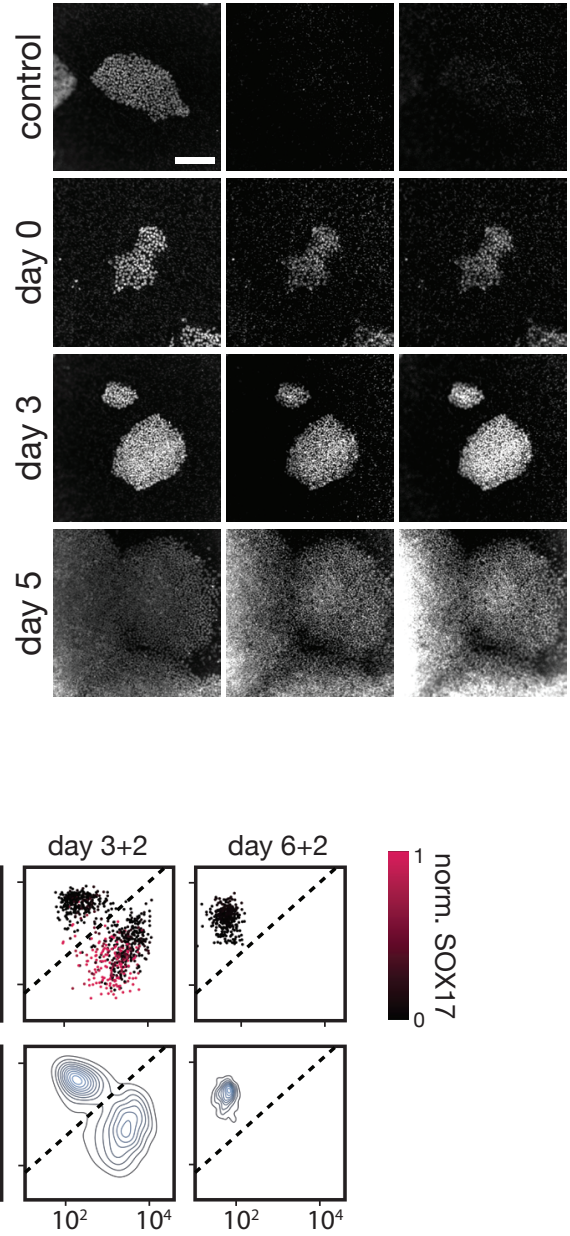

OCT4 (a.u.)

\section{h}
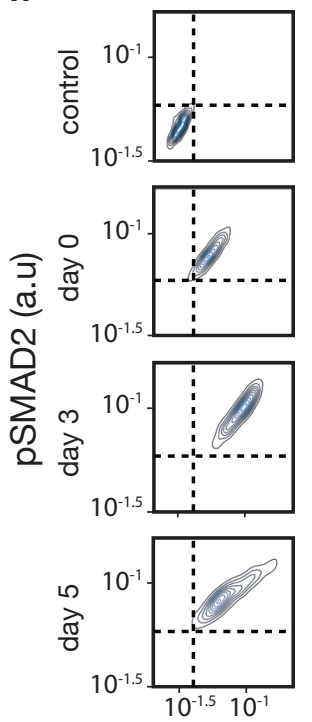

pSMAD1 (a.u.)

Figure S1 
Figure S1: Differentiating stem cells produce mesendoderm-derived and ectoderm-derived cell types while maintaining the ability to receive BMP4 and Activin signals

a, Top, confocal microscopy image of immunostained hESC colony after 48 hours of BMP4 + Activin A; SOX17 (pink), OCT4 (yellow), SOX2 (blue). These cells largely adopt SOX17 ${ }^{+}$ endoderm fates and are uniformly OCT4 ${ }^{+} \mathrm{SOX}_{2}{ }^{-}$. Scale bar $=100 \mu \mathrm{m}$. Bottom Left, contour plot of cell density as a function of OCT4 and SOX2 levels in individual cells; Bottom Right, same plot as left displayed as scatter plot with individual cells colored based on the level of endodermal marker SOX17. b, Images of hESC colonies immunostained for OCT4, SOX2, and mesendoderm marker T after 1-6 days of Activin/NODAL inhibition followed by $42 \mathrm{~h}$ of BMP4 + Activin A stimulation. $\mathrm{T}^{-} \mathrm{OCT}^{-} \mathrm{SOX}^{+}$ectoderm cells first appear at 2 days and are widespread by 4 days of Activin/NODAL inhibition. The spatial structure seen here largely appears after BMP4 and Activin A signal induction and is likely due to a combination of local density impacts on ectoderm-directed differentiation rate and homophilic interactions between cells. Scale bar $=$ $300 \mu \mathrm{m}$. c, Top, epifluorescence microscopy image of an hESC colony immunostained for the neurectoderm marker PAX6 (purple), OCT4 (yellow), and SOX2 (blue) after 6 days of Activin/NODAL inhibition. These cells largely adopt $\mathrm{PAX} 6^{+} \mathrm{OCT}^{-} \mathrm{SOX}^{+}$neurectoderm fates. Below, contour plot of cell density as a function of OCT4 and SOX2 levels in individual cells (left), same plot as left displayed as scatter plot with individual cells colored based on the level of PAX6 (right). Scale bar $=300 \mu \mathrm{m}$. d, Images of hESC colonies immunostained for OCT4, SOX2, and the neurectoderm marker PAX6 after 0-6 days of Activin/NODAL inhibition. PAX6 expression is first seen after 4 days and is widespread by 5 days. Scale bar $=300 \mu \mathrm{m}$. e, hESCs were exposed to a pretreatment of Activin/NODAL inhibition for 1, 3, or 6 days before BMP4+ Activin A signal exposure (left). Confocal microscopy images of immunostained hESCs at the end of the experiment after $48 \mathrm{~h}$ of BMP4 + Activin A treatment (right). Increasing duration of Activin/NODAL inhibition reduced the population's competence to produce $\mathrm{SOX} 17^{+}$endoderm and, more broadly, $\mathrm{OCT}^{+}$(yellow) SOX2 ${ }^{-}$(blue) mesendoderm-derived cell types. Scale bar $=$ $100 \mu \mathrm{m}$. f, Scatter plot (top) and density contour plot (bottom) of cells from E, as a function of their OCT4 and SOX2 levels. Cells in scatter plot colored by level of SOX17. g, Immunofluorescence images acquired via epifluorescence microscopy showing DAPI, phosphorylated SMAD1, and phosphorylated SMAD2 levels in hESC colonies upon stimulation with BMP4 + Activin for 45 mins after 0, 3, or 5 days of Activin/NODAL inhibition.

Unstimulated control shown in top row. All stimulated cells at all time points displayed nuclearlocalized pSMAD1 and pSMAD2 upon BMP4 + Activin A exposure despite the loss of mesendoderm competence that occurs during this window. Scale bar $=300 \mu \mathrm{m}$. $\mathbf{h}$, Density contour plots of cells treated as in $\mathrm{G}$, as a function of pSMAD2/3 and pSMAD1/5 levels determined by antibody staining. Dotted lines drawn for reference. 

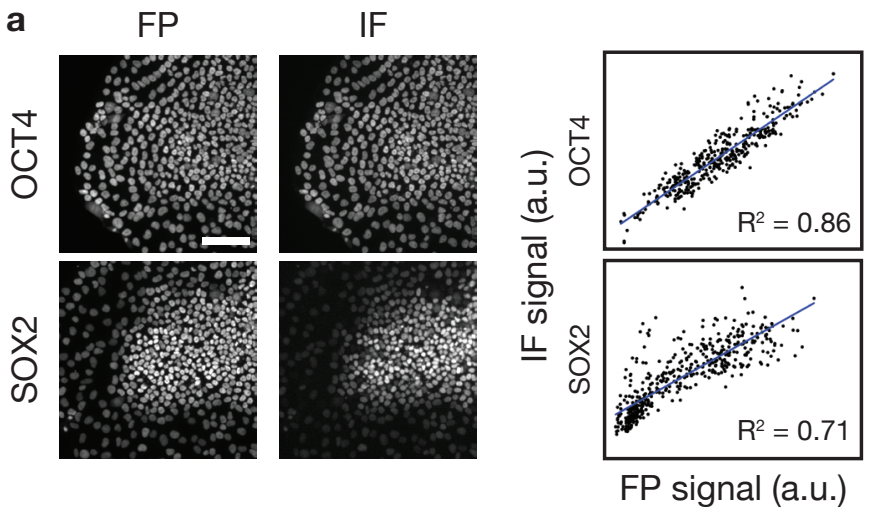

b

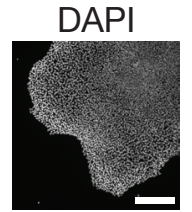

NANOG

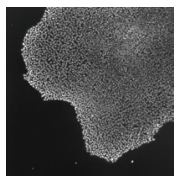

OCT4

SOX2
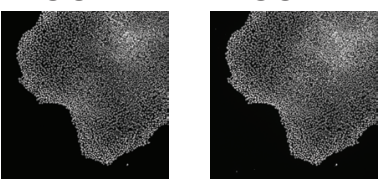

C

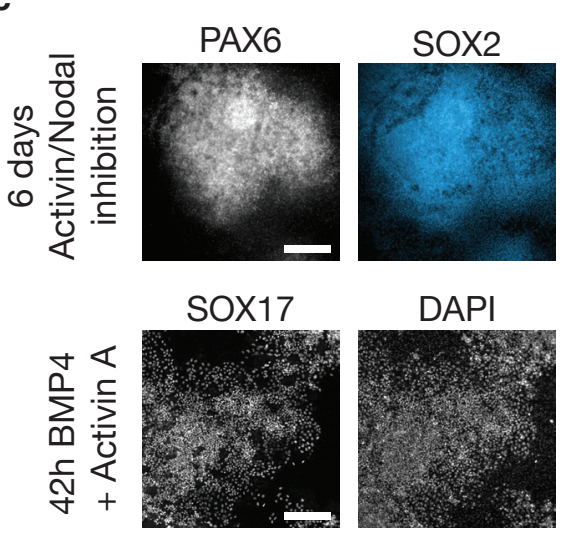

d

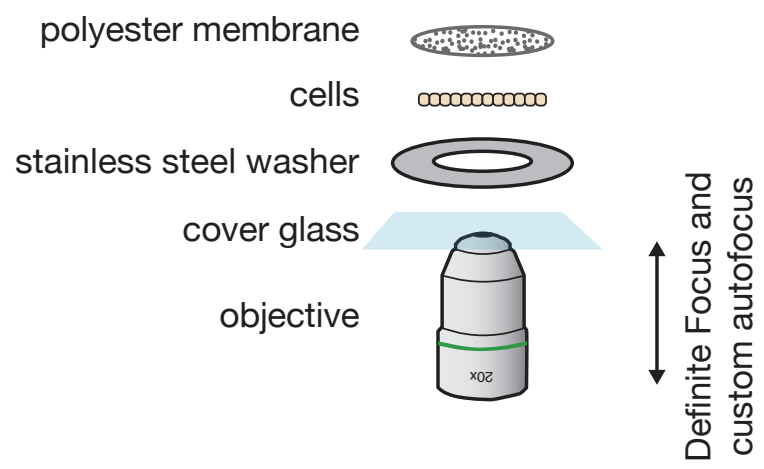

Figure S2 
Figure S2: Monitoring OCT4:RFP and SOX2:YFP accurately reflects OCT4 and SOX2 levels without perturbing cell potential or behavior

a, OCT4 and SOX2 levels as determined by immunofluorescence (IF) and by OCT4:RFP and SOX2:YFP fluorescence (FP). Scale bar $=100 \mu \mathrm{m}$. Blue line, best-fit linear regression. $\mathbf{b}$, Immunofluoresence images of H1 OCT4:RFP SOX2:YFP double reporter cells stained for NANOG, OCT4, and SOX2. Nuclei (DAPI) are shown for reference. These cells can maintain pluripotency indefinitely. Scale bar $=300 \mu \mathrm{m}$. c, Immunofluorescence images of H1 OCT4:RFP SOX2:YFP double reporter cells that were (top) stained for PAX6 and SOX2 after 6 days of Activin/NODAL inhibition by A83-01 or (bottom) stained for SOX17 after 42h of BMP4 and Activin A with nuclei (DAPI) shown for reference. This line's ability to form the germ layers is unaffected. Scale bar $=300 \mu \mathrm{m}$. d, Schematic of the live-cell imaging apparatus constructed for this study. Cells were grown on the bottom of a permeable membrane glued to a thin stainlesssteel washer and imaged from below with an epifluorescence microscope. Focus was maintained with a combination of Zeiss Definite Focus and periodic software autofocus adjustments. 
a

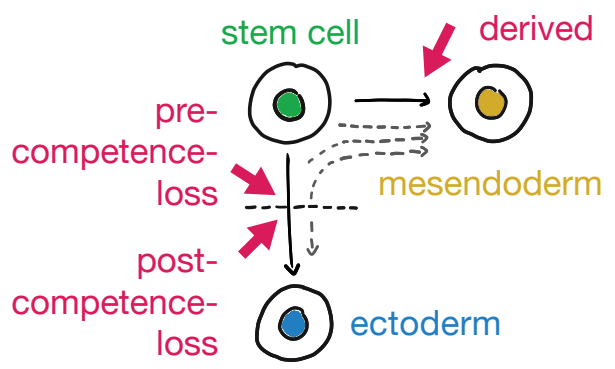

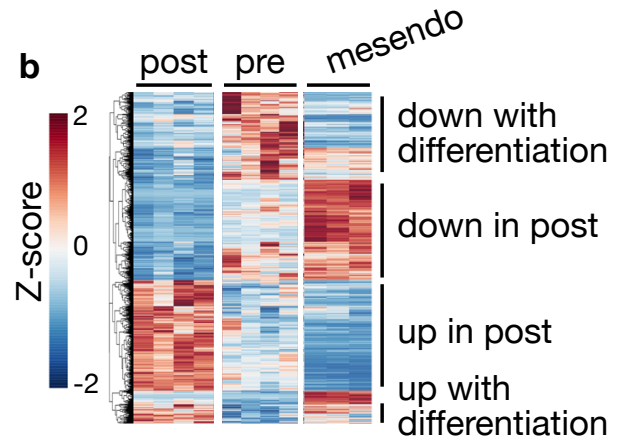

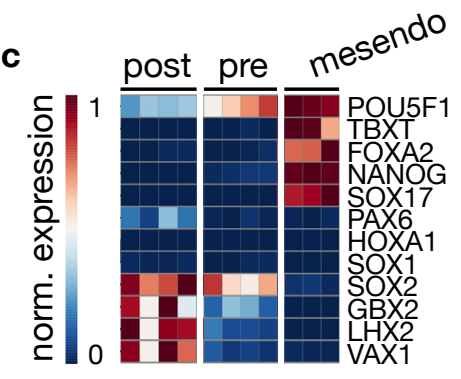

d

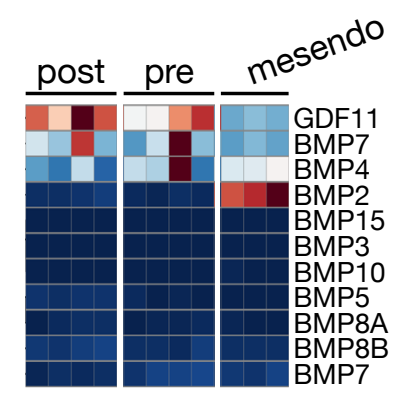

post pre mesendo

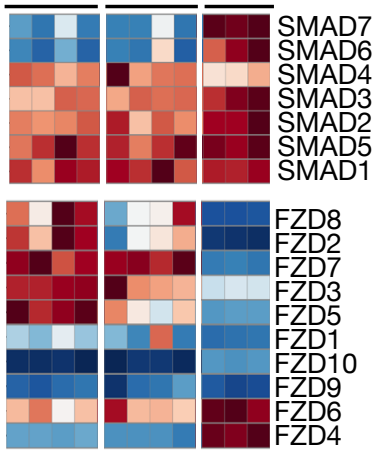

post pre mesendo

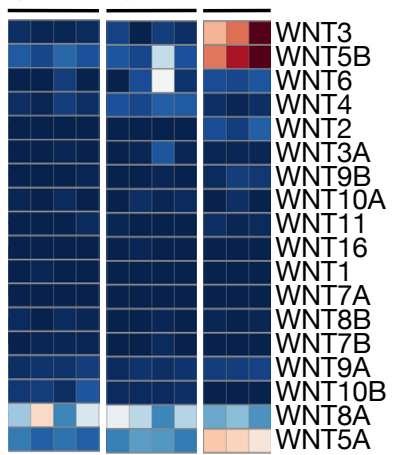

post pre mesendo
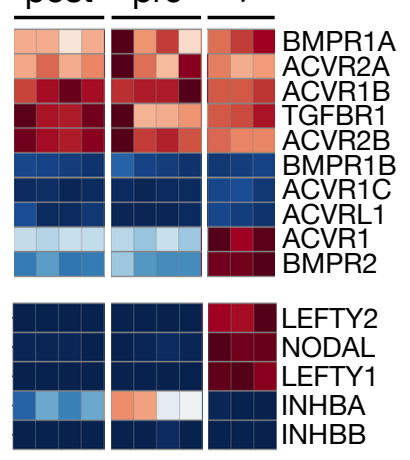

e

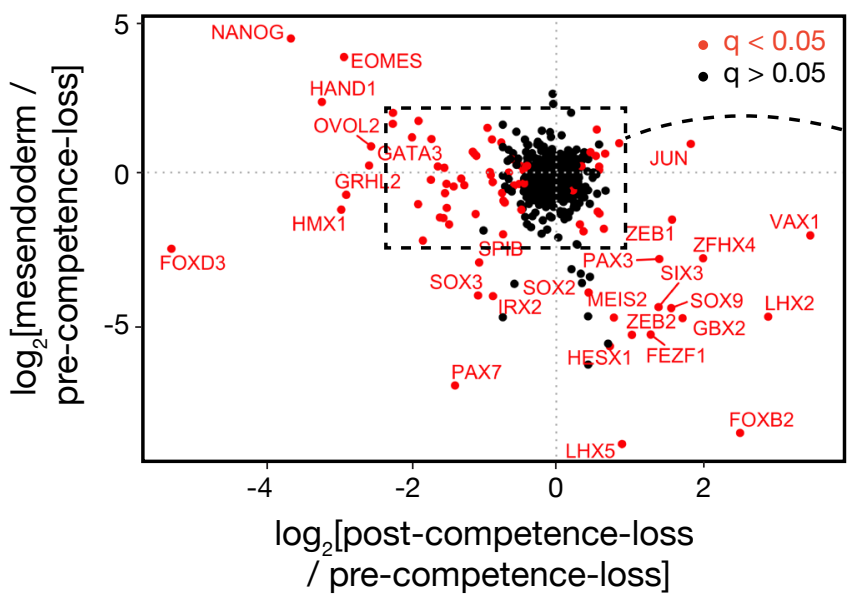

$\mathbf{f}$

epithelial cell morph. involved in placental branching epithelial cell diff. involved in embryonic placenta dev. regulation of timing of catagen
branching involved in labyrinthine layer morphogenesis glomerular visceral epithelial cell development regulation of hair follicle maturation glomerular epithelial development cell diff. involved in embryonic placenta development embryonic plac

fold enrichment
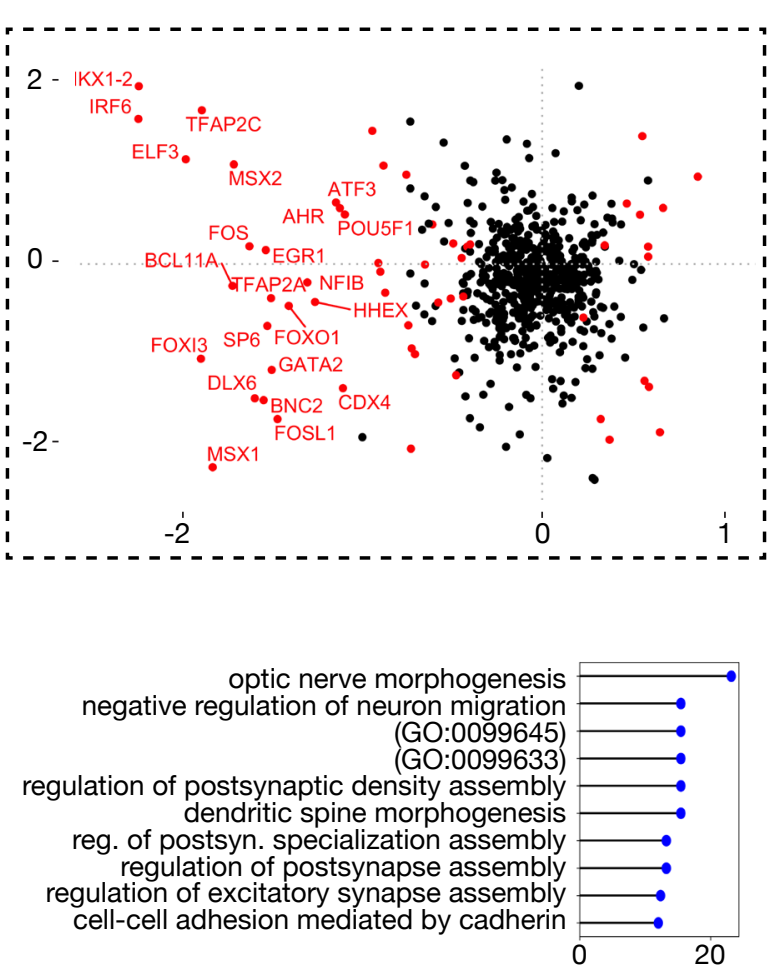

fold enrichment

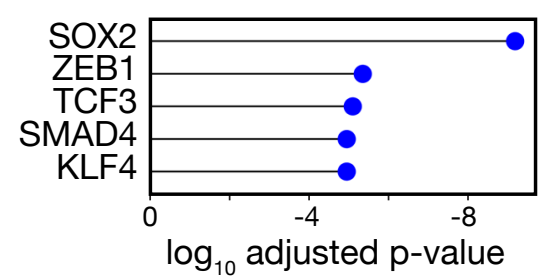




\section{Figure S3: Analysis of gene expression changes that coincide with mesendoderm competence loss}

a, Based on the OCT4:RFP to SOX2:YFP ratio, pre- and post-competence-loss cells were isolated using FACS for RNA-seq and ATAC-seq. Mesendoderm-derived cells (hESCs stimulated with $40 \mathrm{~h}$ of BMP4 + Activin A) were also assayed as an outgroup to allow for the identification of ectoderm lineage-specific changes. b, Heatmap showing normalized gene expression changes for genes that display significant differential expression between at least two of the three assayed populations in (a). c, Heatmap showing normalized expression of selected marker genes whose expression is associated with the early germ layer lineages. Gene expression was normalized by the maximal expression observed in each row or $10 \mathrm{TPM}$, whichever was greater. d, Heatmap showing normalized expression of selected signaling molecules, transducers, and receptors. Gene expression was normalized by the maximal expression observed in each row or 10 TPM, whichever was greater. No significant expression changes between the pre- and postcompetence loss populations were observed in these groups. e, Scatterplot showing log2 fold change for all transcription factors between the (x-axis) post- and pre-competence-loss populations and the (y-axis) mesendoderm and pre-competence-loss populations. TFs that are significantly differentially expressed between the pre- and post-competence-loss populations are shown in red. TFs in the second and fourth quadrants of this plot have lineage-specific expression patterns. f, Ten most significantly enriched GO terms for gene expression sets from this study as calculated by PANTHER. Left, enriched terms for genes that are downregulated upon competence loss in a lineage-specific pattern. Right, same for genes that are upregulated. $\mathbf{g}$, Left, most significant ChIP-seq target set enrichments as reported by Enrichr for genes whose expression was upregulated from pre-competence-loss to post-competence-loss cells but not significantly upregulated from the pre-competence-loss to mesendoderm-derived populations. Right, same for genes that were downregulated from pre- to post-competence-loss but not downregulated from pre-competence loss to mesendoderm populations. 

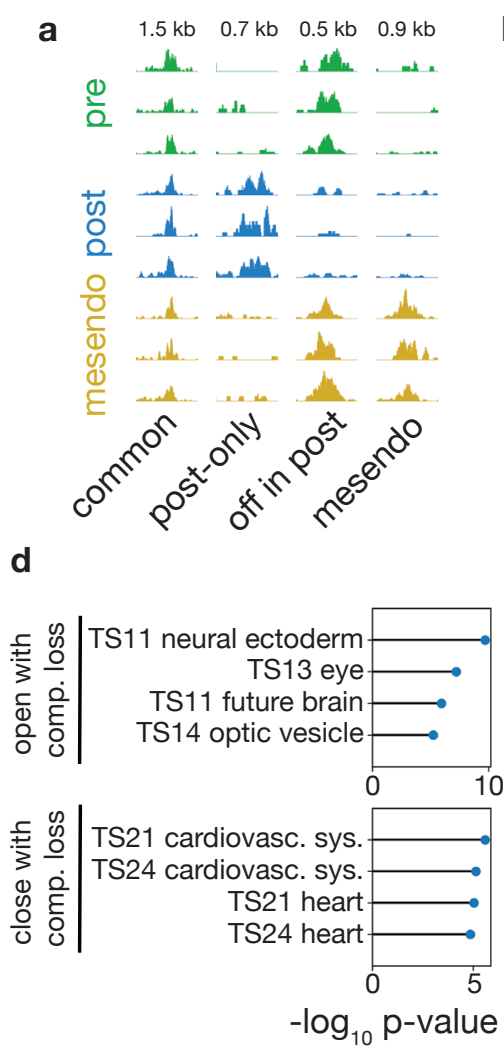
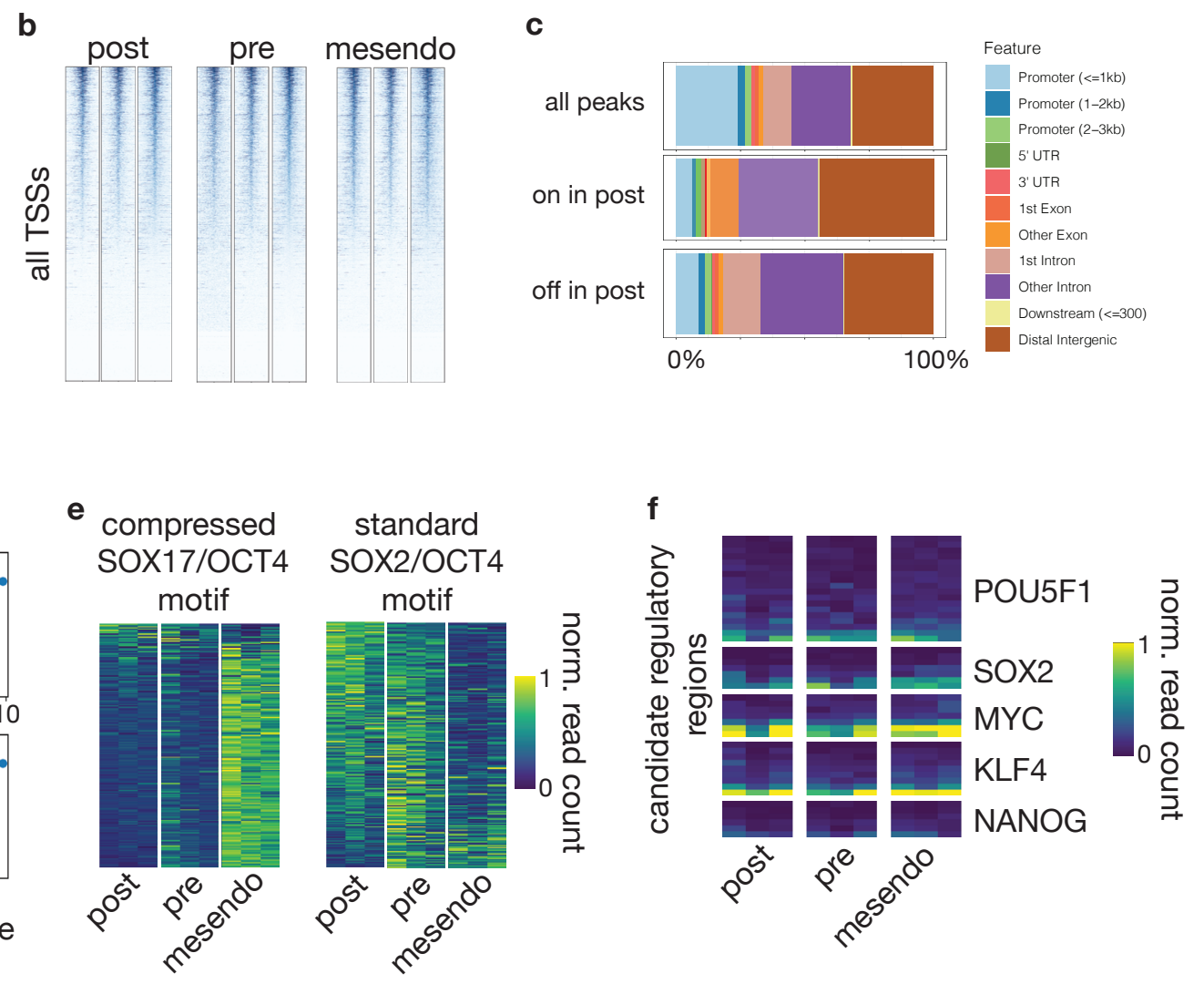

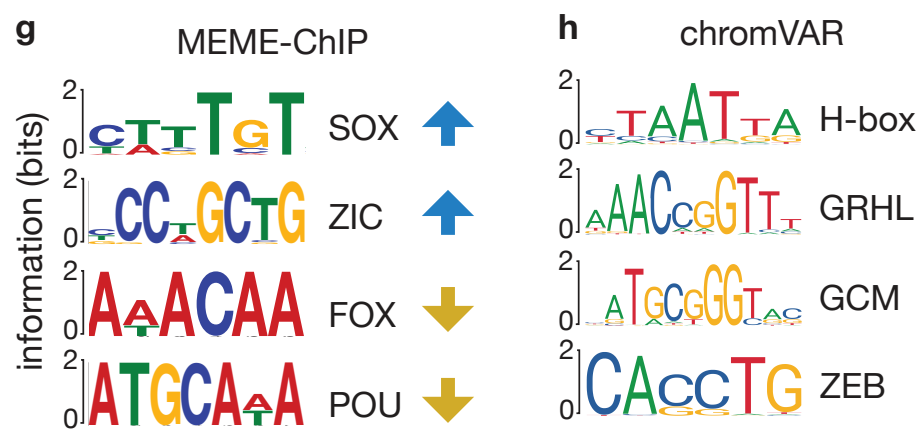

Figure S4 
Figure S4: Analysis of chromatin accessibility changes that coincide with mesendoderm competence loss

a, Normalized ATAC-seq read depth at example loci with the indicated accessibility patterns across pre-competence-loss, post-competence-loss, and mesendoderm-derived populations. Text at top indicates the length of the region shown. b, ATAC-seq read depth at all transcription start sites. Each heatmap block represents all transcription start sites (TSSs) in a single biological replicate for the indicated condition. Each row shows a heatmap representation of read depth in $2 \mathrm{~kb}$ windows centered on each TSS. Rows are sorted for each replicate individually by mean read depth across the window. c, Genomic feature type of the indicated ATAC-seq peaks as annotated by ChIPSeeker. Annotations are shown for all detected peaks across all samples, for peaks that are significantly more accessible in post-competence-loss cells, and for peaks that are significantly less accessible in post-competence-loss cells. Most differentially accessible sites are located in distal intergenic or intronic regions. d, Overlap enrichment of mouse homologs of the differentially expressed gene sets from this study with developmental stage gene expression sets from the Mouse Genome Informatics database as calculated by the software GREAT. Top, the four most enriched gene expression set matches for genes that are upregulated in a lineagespecific manner upon competence loss. Bottom, the same for genes that are downregulated. e, Heatmap showing row-normalized mean ATAC-seq depth in 500 bp regions centered on each peak that shows significant changes in accessibility and which contains the indicated motif. Left, the regions that contain a compressed SOX17/OCT4 motif associated with endoderm occupancy. Right, the same for regions that contain a standard SOX2/OCT4 motif associated with pluripotency occupancy. f, Heatmap showing normalized ATAC-seq read depth in all ENCODEannotated candidate regulatory regions for the indicated pluripotency-associated genes. None of these regions show significant differences between pre- and post-competence-loss cells. $\mathbf{g}$, Selected motifs with significant enrichment in genomic regions that had a significant increase (blue arrows) or decrease (yellow arrows) from the pre-competence-loss to the post-competenceloss populations as discovered using MEME-ChIP. Motifs are labeled by the TF family/group to which they correspond. E-values: SOX, 3.7e-128; ZIC, 8.3e-033; FOX, 1.8e-02; POU, 2.9e-003. h, Top 4 nonredundant motifs that best explain the observed changes in ATAC-seq read depth between pre-competence-loss and post-competence-loss populations, as calculated using chromVAR. p-values: homeobox (VAX2), 9.8E-05; GCM (GCM1), 3.2E-4; GRHL (GRHL1), 6.6E-4; ZEB (ZEB2), 6.9E-4. 
bioRxiv preprint doi: https://doi.org/10.1101/867010; this version posted March 14, 2020. The copyright holder for this preprint (which was not certified by peer review) is the author/funder. All rights reserved. No reuse allowed without permission.

a

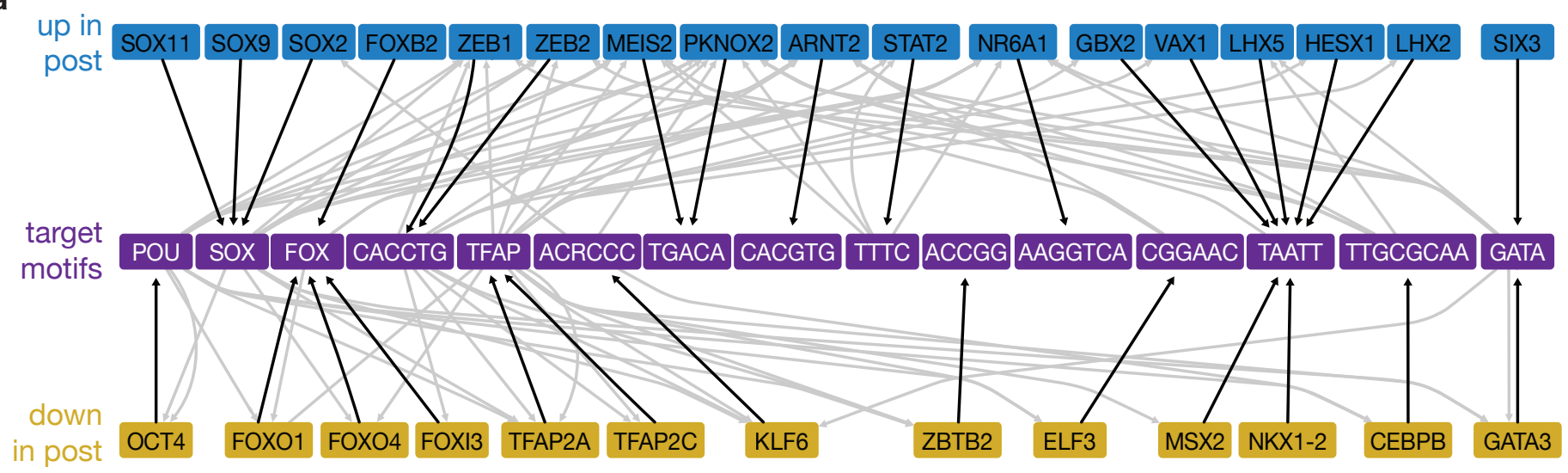

b

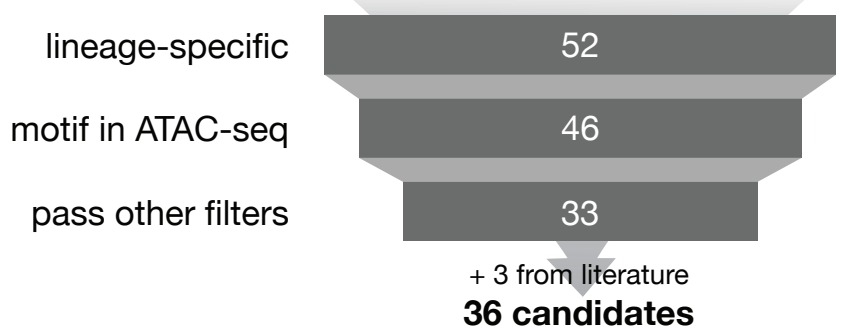

C full list of candidates

$\begin{array}{llll}\text { AHR } & \text { GRHL1 } & \text { NFIB } & \text { SOX2DBD } \\ \text { ARNT2 } & \text { GSC } & \text { NRF2 } & \text { SOX9 } \\ \text { ATF3 } & \text { HESX1 } & \text { OCT4 } & \text { TFAP2A } \\ \text { FEZF1 } & \text { ID2 } & \text { OCT4DBD } & \text { TFAP2C } \\ \text { FOXB2 } & \text { JUNB } & \text { OTX2 } & \text { VAX1 } \\ \text { FOXJ2 } & \text { KLF6 } & \text { PAX3 } & \text { ZSCAN23 } \\ \text { FOX01 } & \text { LHX2 } & \text { PKNOX2 } & \\ \text { FOXO4 } & \text { LHX5 } & \text { POU2F3 } & \\ \text { GATA3 } & \text { MBNL2 } & \text { POU6F1 } & \\ \text { GBX2 } & \text { NANOG } & \text { SOX2 } & \end{array}$


Figure S5: Gene expression and chromatin accessibility signatures reveal a core set of TFs that, when perturbed, could modulate competence

a, Candidate gene regulatory network that governs mesendoderm competence. TFs that are upregulated (top row, blue) or downregulated (bottom row, yellow) specifically between pre- and post-competence-loss populations are connected to their DNA binding motifs by black arrows. Each motif is connected to possible regulatory targets by gray arrows according to the following criteria (1) there is a significant match to that motif in an ATAC-seq peak that is (2) predicted to be associated with the target gene via the CisMapper software. b, Schematic depicting the candidate selection process (see methods for full details). c, Full list of the 36 TFs identified as candidates for perturbation to affect mesendoderm competence 
a

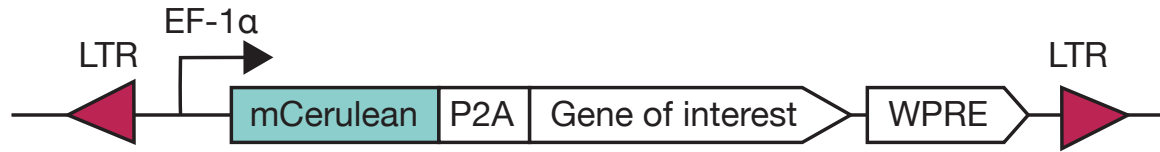

b

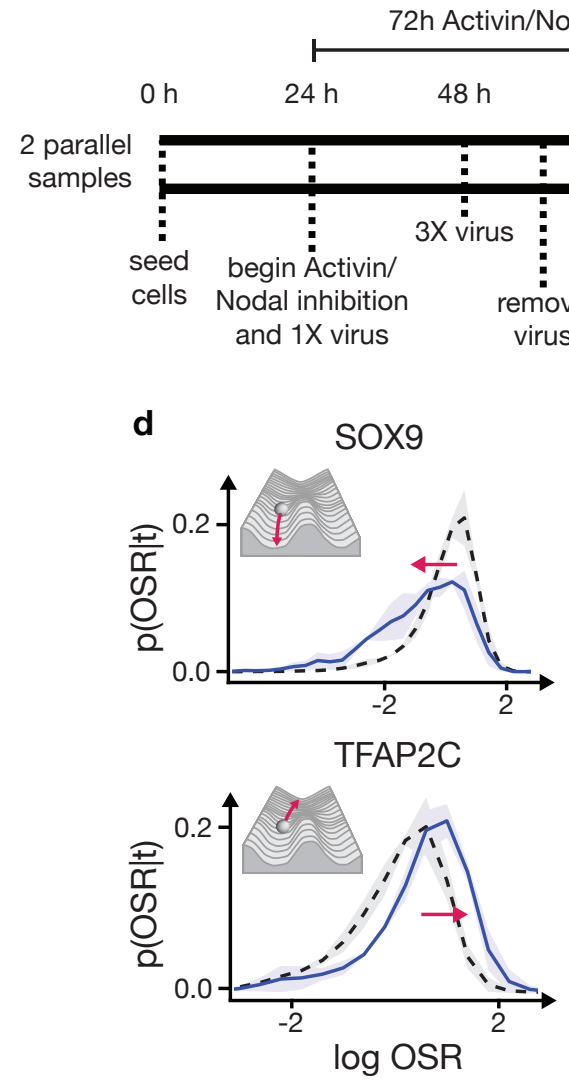

f

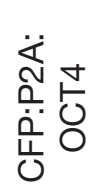

PAX6 / CFP

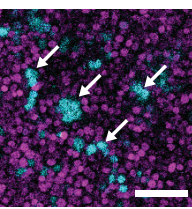

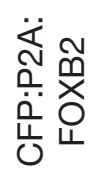
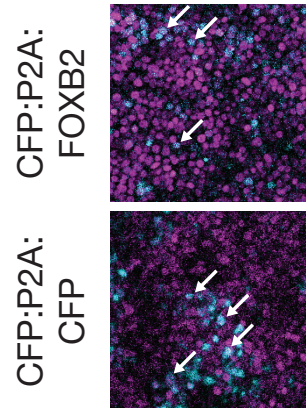

C

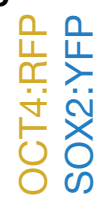

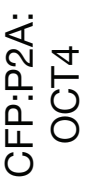

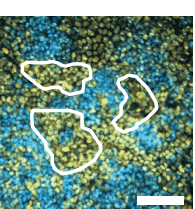

CFP
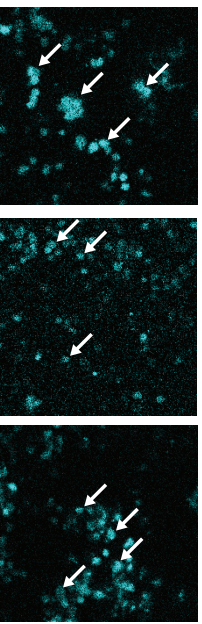

PAX6
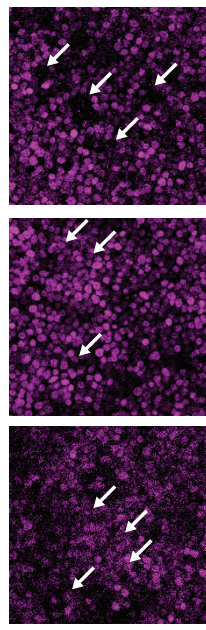

e

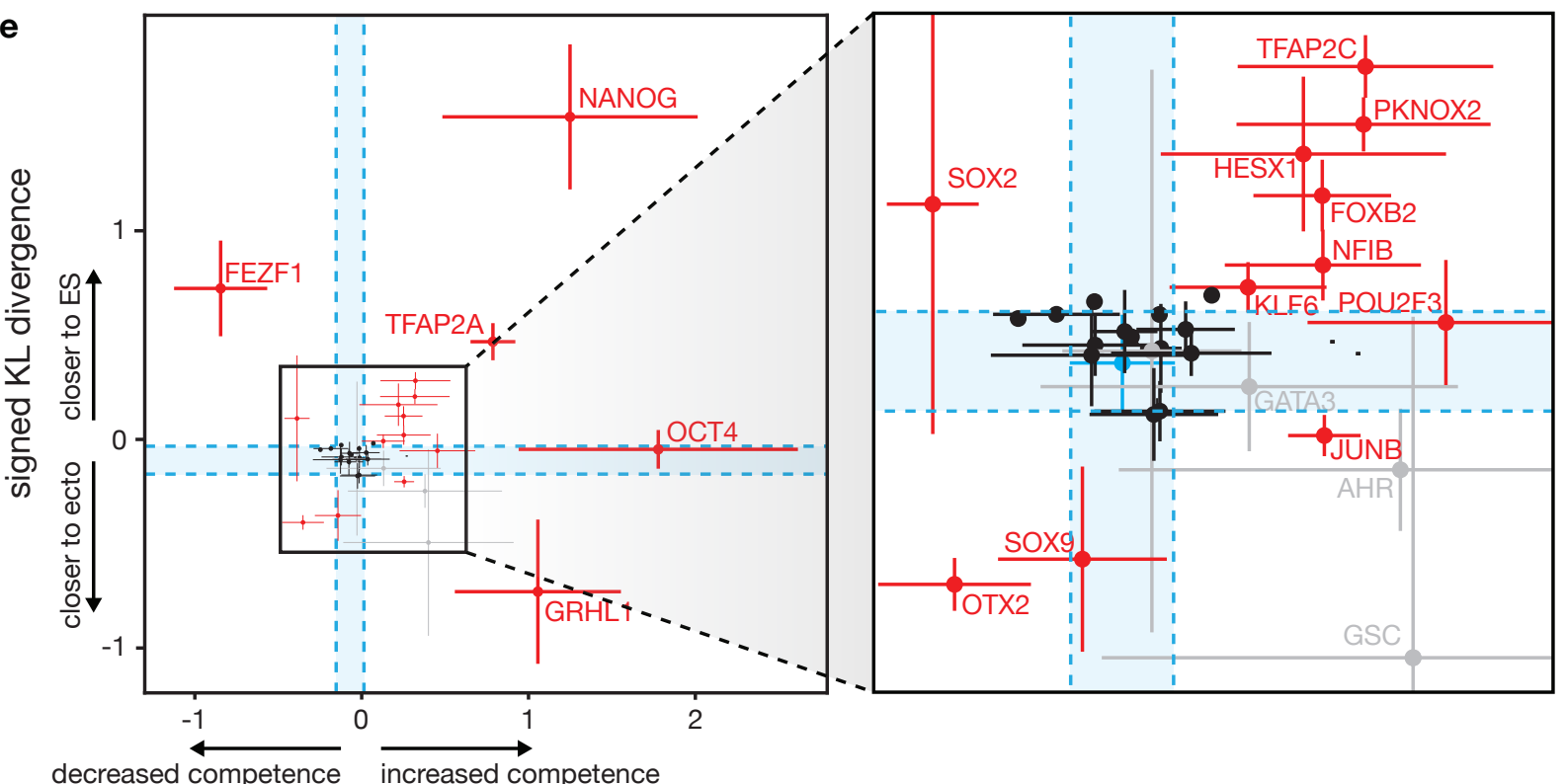

shift in location of $\mathrm{p}$ (mesendoderm|OSR $=0.5$ from non-transduced to transduced populations $\left(\log _{2} \mathrm{OSR}\right)$ 


\section{Figure S6: Analysis of lineage bias and differentiation dynamics in cells perturbed by candidate TFs}

a, Schematic of the cassette transduced via lentiviral vector. Under the control of the EF1alpha promoter, we placed the cyan fluorescent protein mCerulean fused in frame to a P2A ribosomal skip sequence and the gene of interest. A Woodchuck Hepatitis Virus Posttranscriptional Regulatory Element (WPRE) was included to improve transcript stability. The entire sequence was flanked by the Long Terminal Repeat regions (LTR) that the viral transposases recognize to effect integration into the genome. b, Schematic of experimental protocol. $24 \mathrm{~h}$ after seeding in pluripotency maintenance conditions, parallel samples of hESCs were subjected to simultaneous Activin/Nodal inhibition and, for the first 36h, lentiviral transduction of the gene of interest. After $72 \mathrm{~h}$ of Activin/Nodal inhibition, one membrane was analyzed by flow cytometry while the other was stimulated with $42 \mathrm{~h}$ of BMP4 + Activin A before analysis by flow cytometry. c, Epifluorescent microscopy images of a cell colony after Activin/Nodal inhibition and subsequent BMP4 + Activin A stimulation. Cells that were transduced with a CFP:P2A:OCT4 expression cassette expressed CFP and preferentially adopted a OCT4:RFP ${ }^{+} \mathrm{SOX} 2: \mathrm{YFP}^{-}$mesendoderm fate. White regions indicate selected clusters of cells expressing CFP, indicating transduction. Scale bar $=100 \mu \mathrm{m}$. d, Examples of two candidates, SOX9 and TFAP2C, whose overexpression altered the distribution of OCT4:RFP/SOX2:YFP ratios of cells before the signal, $\mathrm{p}(\mathrm{OSR} \mid \mathrm{t})$. OCT4:RFP and SOX2:YFP levels were normalized to an hESC sample measured in the same batch. Black dotted line, wildtype. Blue, transduced. Shaded area represents one standard deviation across biological replicates. Inset, a schematic depicting the corresponding effects on the Waddington developmental landscape. e, Scatterplot of the shift in $\mathrm{p}$ (mesendoderm $\mid \mathrm{OSR}$ ) location versus a metric of disruption of $\mathrm{p}(\mathrm{OSR} \mid \mathrm{t})$ for each TF tested. Disruption of initial differentiation dynamics was measured as the Kullback-Leibler divergence of the distribution of the $\log$ ratio of OCT4:RFP to SOX2:YFP of $\mathrm{CFP}^{+}$cells using the same distribution from $\mathrm{CFP}^{-}$ cells as a reference; this KL-divergence was then reported with a positive sign if the mean OSR of the transduced cells was higher than in the non-transduced cells and a negative sign if not. Candidates that were significantly different from the CFP negative control (FDR $<0.1$ ) along either dimension are shown in red. Candidates whose effects were not consistent across replicates are shown in gray. CFP:P2A:CFP is shown in blue. Blue dotted lines indicate CFP:P2A:CFP overexpression outcomes, shown as the mean \pm one standard deviation of $n=4$ biological replicates, for each axis. OCT1, POU6F1, PAX3 were tested but are excluded from this plot because their overexpression caused premature downregulation of both OCT4:RFP and SOX2:YFP, indicating possible differentiation to an alternative fate. Interestingly, exogenous OCT4 overexpression does not impact the dynamics of endogenous OCT4:RFP and SOX2:YFP, as evidenced by the location of the OCT4 point near zero on the y-axis. $\mathbf{f}$, Immunofluorescence images showing PAX6 (magenta) and CFP (cyan) after 6 days of neurectoderm-directed differentiation using inhibitors of both Activin/NODAL and BMP. Top row, exogenous expression of CFP:P2A:OCT4; Middle row, CFP:P2A:FOXB2; Bottom row, CFP:P2A:CFP. White arrows indicate select CFP-expressing cells. Look-up tables for the channels in each condition are scaled individually because variable membrane positioning in the well created spurious changes in overall apparent signal intensity. Scale bar $=50 \mu \mathrm{m}$. OCT4 overexpression prevents PAX6 induction, while FOXB2 or CFP overexpression does not disrupt normal PAX6 induction. 